\title{
Review Article \\ Recent Advances in Understanding Magnetic Nanoparticles in AC Magnetic Fields and Optimal Design for Targeted Hyperthermia
}

\author{
Hiroaki Mamiya \\ National Institute for Materials Science, Tsukuba 305-0047, Japan \\ Correspondence should be addressed to Hiroaki Mamiya; mamiya.hiroaki@nims.go.jp
}

Received 19 April 2013; Accepted 17 June 2013

Academic Editor: Oleg Petracic

Copyright ( 2013 Hiroaki Mamiya. This is an open access article distributed under the Creative Commons Attribution License, which permits unrestricted use, distribution, and reproduction in any medium, provided the original work is properly cited.

Targeted hyperthermia treatment using magnetic nanoparticles is a promising cancer therapy that enables selective heating of hidden microcancer tissues. In this review, I outline the present status of chemical synthesis of such magnetic nanoparticles. Then, the latest progress in understanding their heat dissipation mechanisms under large magnetic fields is overviewed. This review covers the recently predicted novel phenomena: magnetic hysteresis loops of superparamagnetic states and steady orientations of easy axes at the directions parallel, perpendicular, or oblique to the AC magnetic field. Finally, this review ends with future prospects from the viewpoint of optimal design for efficacy with a low side-effect profile.

\section{Introduction}

Hippocrates said, "those diseases which drugs cannot cure, the knife cures; those which the knife cannot cure, fire cures; those which fire does not cure must be considered incurable." In one respect, medicine has not changed over time; even today, several treatments are used in combination to treat illnesses that have no established effective treatment protocol, the most prominent example being cancer. The current standard treatments for cancer include surgery, chemotherapy, and radiotherapy. Beyond these treatments, much research is being undertaken to create several new treatment options such as immunotherapy, and the modern equivalent of Hippocrates "fire": thermotherapy. Thermotherapy is a treatment method that exploits the lowered heat resistance of cancerous tissues compared with that of normal tissues. Cancerous tissues undergo cell death even at temperatures within the range of 42 to $43^{\circ} \mathrm{C}$, thus rendering thermotherapy as a promising option to reduce the disease burden in a patient [1].

To reduce damage to normal tissues using standard treatments, endo-/laparoscopic surgical techniques have been developed as a modern equivalent of Hippocrates' "knife." For chemotherapy, much effort has focused on drug delivery to selectively transport anticancer agents to tumor tissues using antigen-antibody reactions. Such drug delivery systems are also used to concentrate boron compounds in tumor tissues. In the treatment known as boron neutron capture therapy, the patient is radiated with epithermal neutrons, which selectively induce $\alpha$-decay of boron nuclei concentrated inside hidden tumors, thus specifically destroying cancer cells. Along these lines, could thermotherapy also utilize drug delivery technology to specifically deliver thermal seeds to cancer cells at unknown locations? For example, if it were possible to develop miniature induction-heating cooking pans and selectively send these to hidden tumors, then would this result in selective heating of the tumor tissues in a human body on an induction-heating cooker? In contrast to a microwave oven, we know that placing one's hand over the induction-heating cooker will not immediately result in a burn. (Usually, we cannot confirm this feature using the commercial IH cooker, because it automatically stops working when we take off a metal pan from it.) This experience shows that a radio-waveband AC magnetic field generated in the cooker can easily penetrate deep into tissues where a tumor may be embedded. Therefore, we believe that the simple idea mentioned here, targeted hyperthermia 
using magnetic nanoparticles, has the potential to selectively destroy cancer cells hidden deep in the body [2-8].

Of course, there are many questions about this concept that need to be addressed. Is it safe to put magnetic nanoparticles in the body? Can magnetic nanoparticles really be concentrated in hidden tumor tissues? Can magnetic nanoparticles be heated within the body? To answer these questions, DeNardo et al. injected iron oxide nanoparticles conjugated with monoclonal antibodies into mouse tails and found that they accumulated at a concentration $c$ of approximately $0.3 \mathrm{~kg} / \mathrm{m}^{3}\left(0.3 \mathrm{mg} / \mathrm{cm}^{3}\right)$ in tumors [9]. (The side effects of iron oxide nanoparticles as an MRI contrast agent had been previously studied and approved for intravenous injection.) Wust et al. showed that injecting high concentrations $(c \sim$ $10 \mathrm{~kg} / \mathrm{m}^{3}$ ) of magnetic nanoparticles directly into tumors at known locations and irradiating them with an AC magnetic field caused the temperature of the tumors to increase to $43^{\circ} \mathrm{C}[10]$. Therefore, it is known that iron oxide nanoparticles are safe, can be selectively accumulated in hidden tumors to some degree, and can be adequately heated when existing at high concentrations. Nevertheless, problems with targeted hyperthermia using magnetic nanoparticles are still evident. Does the density of magnetic nanoparticles delivered to tumors increase as it does when they are directly injected into tumors? If not, is it possible to compensate for a lower density of magnetic nanoparticles by maximizing their heat dissipation? The first problem is primarily a biochemical one, so materials researchers have primarily focused on improving the heating performance of magnetic nanoparticles [1130]. Consequent advances in chemical synthesis technology have resulted in the fabrication of magnetic nanoparticles of engineered size, shape, and structure. With respect to physical heating mechanisms, the nature of the nonlinear response and nonequilibrium dissipation in AC magnetic fields of magnetic nanoparticles, which are in contrast to the properties of cooking pans, have been uncovered. This review addresses this progress as follows. In Section 2, conventional models that are the basis of the traditional design of hyperthermia treatments are introduced. In Section 3, advances in the synthesis of magnetic nanoparticles are described and limitations in the conventional models when the monodisperse nanoparticles are used in actual thermotherapy are considered. In Section 4, recent advances in the knowledge of heating mechanisms provided by numerical simulations are explained. Finally, we summarize the optimal design of magnetic nanoparticles for hyperthermia treatment and discuss their potential as an effective and safe version of Hippocrates' "fire" in Section 5.

\section{Conventional Models for Magnetic Response to AC Magnetic Fields [31-34]}

The main advantage of hyperthermia treatment using magnetic nanoparticles is that the nanoparticles can reach the cancer tissue directly by travelling through the submicrometer spaces between blood cell walls. Therefore, for practical use, the nanoparticles should not form long chains or large clusters. Even though the many-body effects caused by dipole-dipole coupling $J_{\mathrm{dd}}$ are not fully understood [3538], it is known that a dispersion becomes unstable if $J_{\mathrm{dd}}$ between the closest nanoparticles is more than five times the thermal energy $[35,38]$. Under these conditions, the minimum allowable distance between iron nanoparticles with diameter $d$ of $12 \mathrm{~nm}$ is roughly $27 \mathrm{~nm}$, and that between ferrite nanoparticles with $d$ of $25 \mathrm{~nm}$ is almost $40 \mathrm{~nm}$. In contrast, small-angle neutron scattering experiments have indicated that the thickness of an absorbed layer is normally several nanometers [39]. Therefore, the upper limit of $d$ is estimated to be roughly $12 \mathrm{~nm}$ for iron and $25 \mathrm{~nm}$ for ferrite. These values would be references for considering the criteria for easy delivery of the nanoparticles, although agglomeration, aggregation, or flocculation may occur depending on the surface charge of biofunctionalized nanoparticle or on the interaction between tumor-targeting ligands. Note that these values are smaller than the critical diameters for the transition into a single-domain configuration and for the coherent reversal of all spins [40]. Therefore, it has been considered that a magnetic nanoparticle used in hyperthermia treatment has only one magnetic moment, $\boldsymbol{\mu}=\mathbf{M}_{s} V$, where $\mathbf{M}_{s}$ is the spontaneous magnetization and $V=\pi / 6 \cdot d^{3}$ is the volume of the magnetic core of the nanoparticle. Such magnetic nanoparticles have been conventionally classified as "ferromagnetic" or "superparamagnetic," depending on whether the direction of $\boldsymbol{\mu}$ thermally fluctuates or not.

Firstly, a ferromagnetic nanoparticle with uniaxial magnetic anisotropy, anisotropy constant $K$, is considered, where $V$ is large enough that its magnetic anisotropy barrier with a height of $K V$ blocks the thermal fluctuations; accordingly, the remanent state appears to be permanent [41]. If a magnetic field $\mathbf{H}$ is applied in the direction antiparallel to $\boldsymbol{\mu}$, the state becomes metastable, as depicted in Figure 1(a). Then, $\boldsymbol{\mu}$ reverses when the barrier disappears at the anisotropy field $H_{K}=2 K /\left(\mu_{0} M_{s}\right)$; consequently, the Zeeman energy falls from $\mu_{0} \mu H_{K}$ to $-\mu_{0} \mu H_{K}$ and the energy corresponding to the difference dissipates, where $\mu_{0}$ is permeability of vacuum. In this case, the work done in one cycle of the AC magnetic field $H_{\mathrm{ac}} \sin (2 \pi f \cdot t)$ is 0 for $H_{\mathrm{ac}}<H_{K}$ and $4 \mu_{0} \mu H_{K}$ for $H_{\mathrm{ac}}>H_{K}$. This kind of heat dissipation has been termed "hysteresis loss." Briefly, the heat dissipation from nanoparticles with unit weight during unit time, also called specific loss power $P_{H}$, abruptly increases from zero to $4 \mu_{0} \mu H_{K} \cdot f \cdot w^{-1}$ (= $\left.4 \mu_{0} M_{s} H_{K} \cdot f \cdot \rho^{-1}\right)$ when $H_{\mathrm{ac}}$ becomes higher than $H_{K}$, where $w$ and $\rho^{-1}$ are the weight and density of the magnetic core of the nanoparticles, respectively. Then, $P_{H}$ flattens out even if $H_{\mathrm{ac}}$ is strengthened further. According to this argument, the guiding principle for maximizing $P_{H}$ of ferromagnetic nanoparticles is that $H_{\mathrm{ac}}$ is adjusted to $H_{K}$ and the number of cycles $f$ is maximized.

Next we move to smaller superparamagnetic nanoparticles with thermally fluctuating reversal of $\boldsymbol{\mu}$ [42]. The reversal probability in a zero magnetic field is expressed as

$$
\tau_{N}^{-1}=f_{0} \cdot \exp \left(\frac{-K V}{k_{B} T}\right),
$$

where $\tau_{N}$ is the Néel relaxation time, $f_{0}$ is the attempt frequency of $10^{9} \mathrm{~s}^{-1}, k_{B}$ is the Boltzmann constant, and $T$ is 


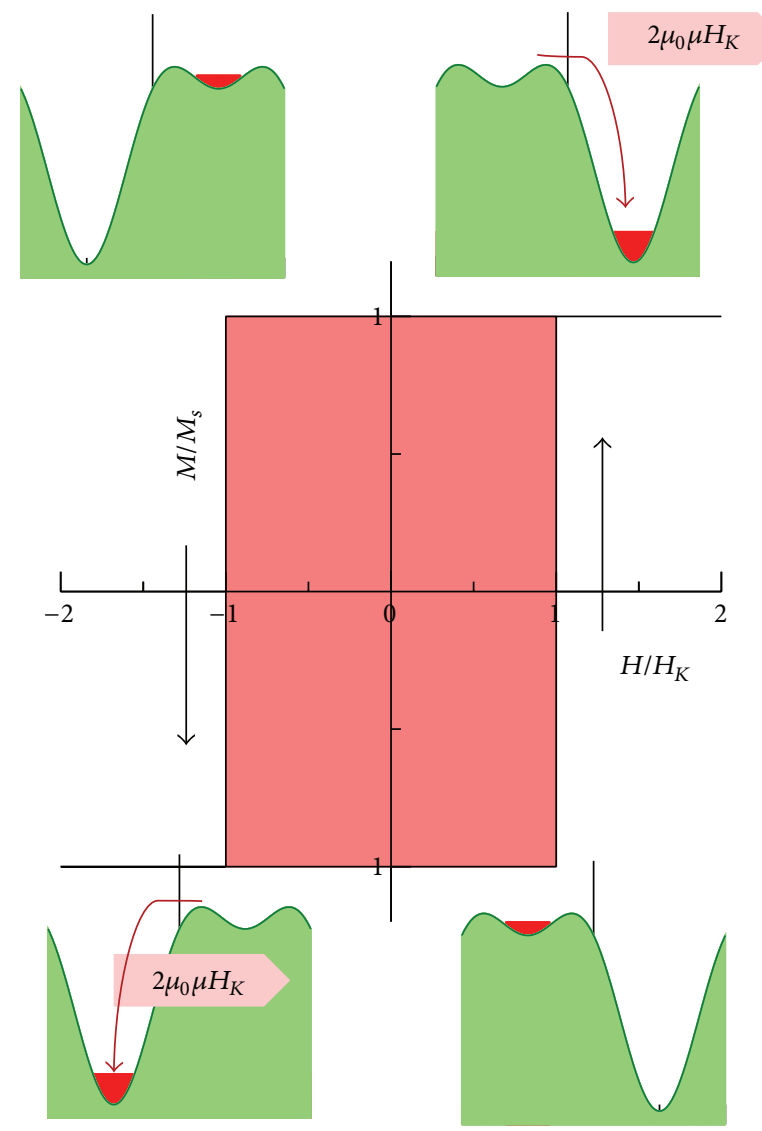

(a) Hysteresis loss

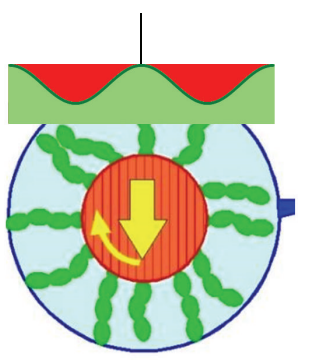

Néel relaxation

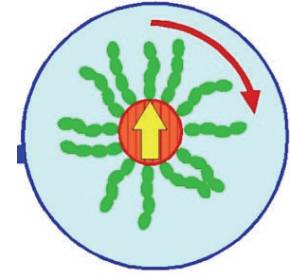

Brownian relaxation

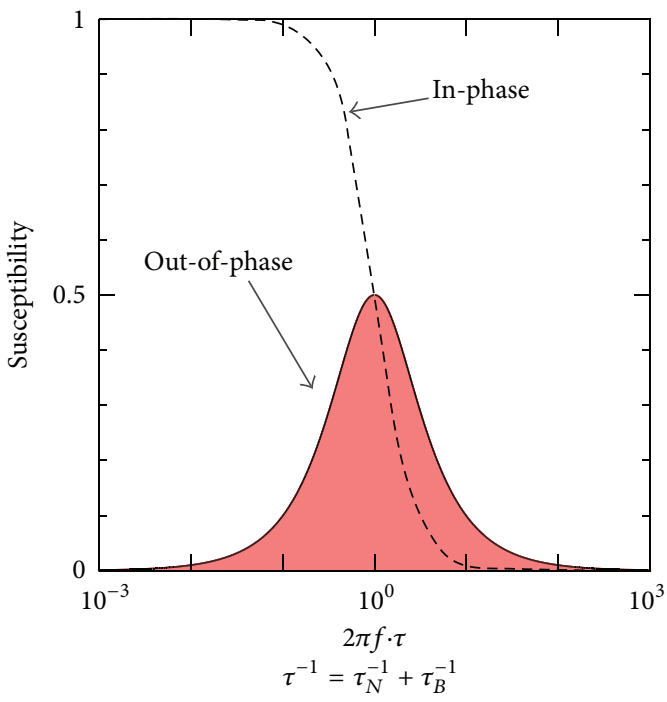

(b) Relaxation loss

FIGURE 1: Schematic diagrams of conventional models for magnetic loss. (a) Hysteresis loss equivalent to the area of $M-H$ loop, and the potential energy in magnetic fields. (b) Relaxation loss given by the out-of-phase component of AC susceptibility. As illustrated in the sketches, in Néel relaxation, the magnetic moment shown by the yellow arrow reverses (the particle does not rotate), while in Brownian relaxation, the magnetic core (the red sphere) rotates with absorbed molecules (the green chains) as a whole (the magnetic moment does not reverse).

the temperature. We must also consider Brownian rotation of the nanoparticles if they are dispersed in a liquid phase. In this case, the characteristic time of the rotation, Brownian relaxation time, in a zero magnetic field is given by

$$
\tau_{B}=\frac{3 \eta V_{H}}{\left(k_{B} T\right)}
$$

where $\eta$ is the viscosity of the liquid phase and $V_{H}$ is the hydrodynamic volume of the nanoparticles including surface modification layers. If reversal and rotation occur in parallel, the characteristic time of relaxation $\tau$ could be expressed as the following equation:

$$
\tau^{-1}=\tau_{N}^{-1}+\tau_{B}^{-1}
$$

For very small superparamagnetic nanoparticles, $\tau$ is determined only by $\tau_{N}$ because $\tau_{N}^{-1}$ increases exponentially with decreasing $V$, while the increase of $\tau_{B}^{-1}$ is inversely proportional to $V_{H}$.

If a linear response of the thermodynamic equilibrium state of such nanoparticles is assumed for small $H_{\mathrm{ac}}$, the average out-of-phase component of AC susceptibility, $\chi^{\prime \prime}$, contributed from each nanoparticle is given by

$$
\chi^{\prime \prime}=\frac{\mu_{0} \mu^{2}}{\left(3 k_{B} T\right)} \cdot \frac{2 \pi f \cdot \tau}{\left[1+(2 \pi f \cdot \tau)^{2}\right]} .
$$

Consequently, "relaxation loss" occurs and its heat dissipation $P_{H}$ is expressed as

$$
\begin{aligned}
P_{H} & =\pi \mu_{0} \chi^{\prime \prime} \cdot H_{\mathrm{ac}}^{2} \cdot f \cdot w^{-1} \\
& =\frac{1}{2} \frac{\left[\mu_{0}^{2} M_{\mathrm{s}}^{2} V /\left(3 k_{\mathrm{B}} T \tau \rho\right)\right] \cdot H_{\mathrm{ac}}^{2} \cdot(2 \pi f \cdot \tau)^{2}}{\left[1+(2 \pi f \cdot \tau)^{2}\right]} .
\end{aligned}
$$

Equation (5) indicates that $P_{H}$ increases in proportion to $f^{2}$ in the low frequency range, $2 \pi f \ll \tau$, whereas it flattens out at $1 / 2\left[\mu_{0}^{2} M_{s}^{2} V /\left(3 k_{B} T \tau \rho\right)\right] \cdot H_{\text {ac }}^{2}$ even if $f$ is increased further in the high frequency range, $2 \pi f \gg \tau$. According to this argument, the guiding principle for maximizing $P_{H}$ of superparamagnetic nanoparticles is that $f$ is adjusted to $\tau^{-1}$ and $H_{\mathrm{ac}}$ is maximized. 


\section{Progress in Synthesis of Magnetic Nanoparticle and Their Use in Thermotherapy}

3.1. Size-, Shape- and Composite-Controlled Synthesis of Magnetic Nanoparticles. As discussed above, to improve hysteresis loss, ferromagnetic nanoparticles with an anisotropy field $\left(H_{K}=2 K /\left(\mu_{0} M_{s}\right)\right)$ matching the amplitude $H_{\mathrm{ac}}$ of the AC magnetic field generated in the oscillator of realistic medical equipment need to be synthesized. In contrast, increasing relaxation loss involves the synthesis of superparamagnetic nanoparticles that have $\tau_{N}$ matching $f$ of the AC magnetic field. For these reasons, a large number of studies have focused on controlling the size, shape, or composite structure of nanoparticles to optimize $H_{K}$ and $\tau_{N}$.

The history of colloids (magnetic fluids) stably dispersing magnetic nanoparticles in solution goes back to the 1960 s, when magnetic suspensions were prepared by pulverizing bulk iron oxide and used for fuel delivery in a weightless environment [43], such as those involving NASA expeditions. Elsewhere, Sato of Tohoku University created magnetic fluids from minute iron oxide particles using chemical methods [44]. There have also been several major subsequent advances in magnetic fluid development, such as the monodisperse iron nitride-based magnetic fluids developed by Nakatani et al. [45]; however, because the industrial applications of magnetic fluids at that time did not require precise control of size, shape, or structure, more extensive research was not conducted in this field. However, in 2000, Sun et al. from IBM described an ordered self-assembled film of monodisperse iron-platinum nanoparticles that could serve as an ultrahigh-tech magnetic recording medium [46]. Since then, researchers have focused on developing methods to synthesize well-controlled nanoparticles, which have been reviewed extensively $[47,48]$. Next, we briefly summarize these methods.

Generally, formation of nanoparticles starts with nucleation in a supersaturated melt, solution, or vapor. Particle growth continues until the concentration of solute atoms falls below the saturation solubility. If nucleation and growth proceed in parallel, nanoparticles formed initially have already grown when the last nanoparticle is formed, thus resulting in nanoparticles of variable size. Furthermore, processes such as coarsening and aggregation simultaneously occur in many cases. One way to obtain monodisperse nanoparticles is the two-stage growth method: in the first stage, rapid heating causes fast supersaturated-burst nucleation, and in the second stage, the gradual precipitation of solute atoms at a temperature below the critical point of supersaturation allows only the existing nanoparticles to grow slowly. In this process, surfactants are often introduced to the solution to prevent coarsening and aggregation. Because all nanoparticles follow the same growth process in this method, their size after growth should in principle be uniform. In practice, different groups have developed particular methods to produce nanoparticles of specific composition and size.

With respect to controlling the shape of a nanoparticle, growth kinetics is essential in addition to thermodynamic stability to minimize surface free energy. For example, if the growth rate for cubic $\{111\}$ surfaces is slower than for $\{100\}$ surfaces, the surface area of $\{100\}$ facets will decrease with growth, and the particles finally become octahedrons of $\{111\}$ facets only. Similarly, if the growth of $\{001\}$ surfaces in a hexagonal crystal system is fast, rods or, conversely, plates can be formed. For this reason, the adsorption of surfactants on particular surfaces has been intensively studied to fabricate a desired shape by controlling the growth rate of each surface. Figure 2 shows examples of regular octahedral and cubic nanoparticles $[49,50]$. With regard to compositing, nanoparticles dispersed in solution are regularly conjugated by substances such as surfactants to lower their surface energy or prevent aggregation, forming a kind of composite material. Advanced compositing techniques have been developed to protect easily oxidized metal cores or to enable the simultaneous expression of multiple functions. For example, dumbbellshaped junctions in different kinds of nanoparticles [51] and core-shell structures [27] have been produced recently (Figure 3).

\subsection{Magnetic Nanoparticles to Maximize Heat Dissipation.} Using these advanced synthesis techniques, researchers have fabricated magnetic nanoparticles to maximize heat dissipation based on the guiding principles described above. As an example, I highlight the recent report by Lee et al. [27], who fabricated novel superparamagnetic nanoparticles with a uniform diameter $d$ of $15 \mathrm{~nm}$ (see Figure 3 again). One of the reasons why they chose such a size may be to avoid aggregation. In addition, the oscillator of their equipment can generate an AC magnetic field of frequency $f=500 \mathrm{kHz}$. As discussed above, superparamagnetic nanoparticles that have a Néel relaxation time $\left(\tau_{N}\right)$ that matches $f$ are required to maximize relaxation loss. Briefly, $\tau_{N}$ should be $(2 \pi f)^{-1}=$ 318 ns. (Overall, $\tau$ needs to be set to $400 \mathrm{~ns}$ when also considering the Brownian relaxation time $\tau_{B}=1.6 \mu \mathrm{s}$.) Substituting $\tau_{N}=318 \mathrm{~ns}$ in (1), the required energy barrier height $(K V)$ is calculated to be $2.4 \times 10^{-20} \mathrm{~J}$. This value corresponds to a uniaxial anisotropic particle with $d=15 \mathrm{~nm}$ and $K=1.4 \times 10^{4} \mathrm{~J} / \mathrm{m}^{3}$. However, examination of parameters such as bulk crystalline magnetic anisotropy [40] revealed that no suitable candidate substances had been reported. For substances with cubic symmetry, the magnitude of $K_{1}$ and the barrier height, $-(1 / 12) K_{1} V$ for negative $K_{1}$ or $(1 / 4) K_{1} V$ for positive $K_{1}$, calculated using $d=15 \mathrm{~nm}$ are as follows: $-1.2 \times 10^{4} \mathrm{~J} / \mathrm{m}^{3}$ and $0.18 \times 10^{-20} \mathrm{~J}\left(\mathrm{Fe}_{3} \mathrm{O}_{4}\right) ;-0.46 \times 10^{4} \mathrm{~J} / \mathrm{m}^{3}$ and $0.08 \times 10^{-20} \mathrm{~J}\left(\gamma-\mathrm{Fe}_{2} \mathrm{O}_{3}\right) ;-0.25 \times 10^{4} \mathrm{~J} / \mathrm{m}^{3}$ and $0.04 \times$ $10^{-20} \mathrm{~J}\left(\mathrm{MnFe}_{2} \mathrm{O}_{4}\right)$; and $18 \times 10^{4} \mathrm{~J} / \mathrm{m}^{3}$ and $8.0 \times 10^{-20} \mathrm{~J}$ $\left(\mathrm{CoFe}_{2} \mathrm{O}_{4}\right)$. As a result, shape control, which affects shape and surface magnetic anisotropy, or composition control or composite structure control, which influences the crystalline magnetic anisotropy, are therefore required. From among the possibilities mentioned, Lee et al. selected core-shell structures of cobalt and manganese ferrites and used a coreand-shell exchange coupling to control the magnitude of effective magnetic anisotropy. As a result, they obtained the core-shell structure shown in Figure 3, with a measured magnetic anisotropy constant $K$ of $1.7 \times 10^{4} \mathrm{~J} / \mathrm{m}^{3}$ (Table 1 ). 

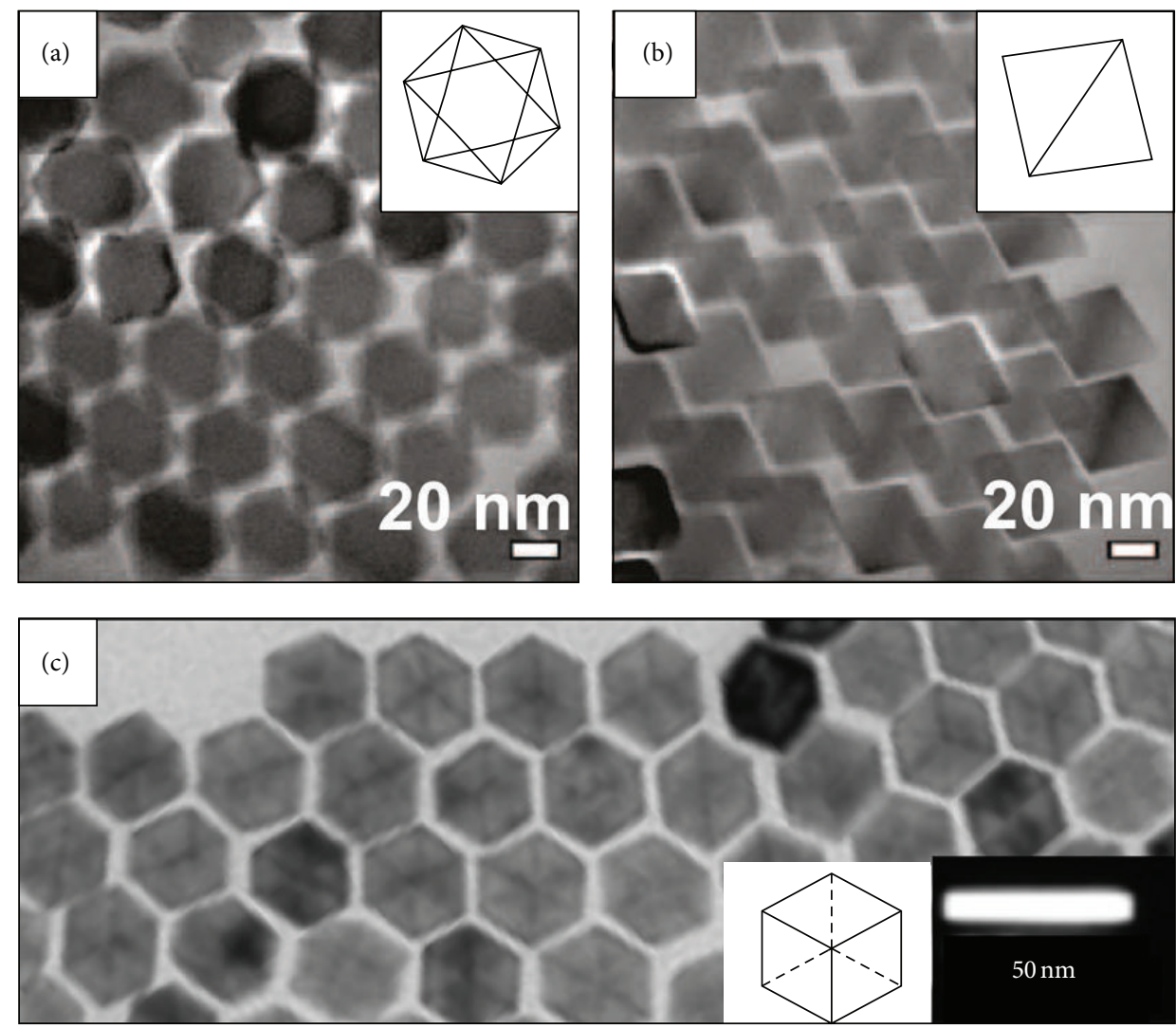

FIGURE 2: Transmission electron micrographs of shape-controlled magnetic nanoparticles with different projection shapes: (a) hexagonal outlines of octahedron-shaped $\mathrm{Fe}_{3} \mathrm{O}_{4}$ nanoparticles (zone axis: $\langle 111\rangle$ ), and (b) parallelogram outlines of the same $\mathrm{Fe}_{3} \mathrm{O}_{4}$ nanoparticles as in (a) (zone axis: $\langle 110\rangle) .{ }^{*}$ Reproduced from Li et al. [49] with permission (Copyright 2010 American Chemical Society). (c) Hexagonal outlines of cube-shaped Ni-Pt nanoparticles (zone axis: $\langle 111\rangle$ ). Private communication (Copyright $2011 \mathrm{~B}$. Jeyadevan).

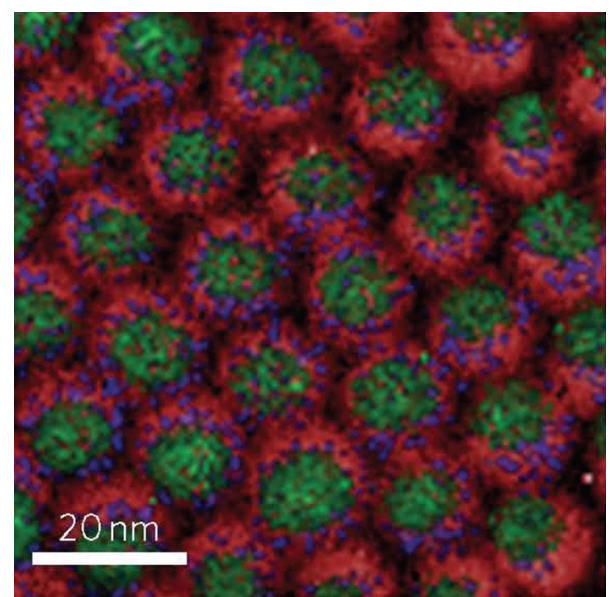

Figure 3: Electron energy-loss spectroscopy (EELS) mapping analysis of $\mathrm{CoFe}_{2} \mathrm{O}_{4} @ \mathrm{MnFe}_{2} \mathrm{O}_{4}$ nanoparticles, where Co, Fe, and $\mathrm{Mn}$ atoms are indicated as green, red, and blue, respectively. ${ }^{*}$ Reproduced from Lee et al. [27] with permission (Copyright 2011 Nature).

When these core-shell nanoparticles were irradiated with an AC magnetic field of frequency $f=500 \mathrm{kHz}$ and amplitude $H_{\mathrm{ac}}=37.3 \mathrm{kA} / \mathrm{m}$, the heat dissipation $\left(P_{H}\right)$ per unit weight reached $3 \mathrm{MW} / \mathrm{kg}(3 \mathrm{~kW} / \mathrm{g})$, which was significantly higher than that using nanoparticles of cobalt ferrite $(0.4 \mathrm{MW} / \mathrm{kg})$
TABLE 1: Size, saturation magnetization $\left(M_{s}\right)$, anisotropy constant $(K)$, and heat dissipation rate per unit weight $P_{H}$ (at $H_{\mathrm{ac}}=$ $37.3 \mathrm{kA} / \mathrm{m}, f=500 \mathrm{kHz}$ ) of ferrite nanoparticles experimentally determined in [27].

\begin{tabular}{lcccc}
\hline Sample & $\begin{array}{c}\text { Size } \\
(\mathrm{nm})\end{array}$ & $\begin{array}{c}M_{s} \\
(\mathrm{kA} / \mathrm{m})\end{array}$ & $\begin{array}{c}K \\
\left(\mathrm{~kJ} / \mathrm{m}^{3}\right)\end{array}$ & $\begin{array}{c}P_{H} \\
(\mathrm{MW} / \mathrm{kg})\end{array}$ \\
\hline $\mathrm{CoFe}_{2} \mathrm{O}_{4}$ & 12 & 510 & 200 & 0.4 \\
$\mathrm{MnFe}_{2} \mathrm{O}_{4}$ & 18 & 700 & 3 & 0.2 \\
$\mathrm{MnFe}_{2} \mathrm{O}_{4} @ \mathrm{CoFe}_{2} \mathrm{O}_{4}$ & 15 & 570 & 17 & 3.0 \\
\hline
\end{tabular}

or manganese ferrite $(0.2 \mathrm{MW} / \mathrm{kg})$. The heat generation of these core-shell nanoparticles is unprecedented so they have received widespread attention.

This example suggests optimized design of nanoparticle synthesis has succeeded in producing nanoparticles that generate large amounts of heat. However, further consideration revealed two notable points. First, the actual amplitude of $H_{\mathrm{ac}}$ reached $37.3 \mathrm{kA} / \mathrm{m}$ or $80 \%$ that of the anisotropic magnetic field, $H_{\mathrm{K}}=2 \mathrm{~K} / M_{s}=47.3 \mathrm{kA} / \mathrm{m}$. This is large enough for the energy barrier to magnetization reversal to disappear because of the Zeeman energy in cases where the direction of the AC magnetic field is not completely parallel to the easy axis of nanoparticles. Thus, these conditions do not permit the application of the guiding principles 
given in (2)-(5) because these assume a linear response for superparamagnetic nanoparticles in zero magnetic field limit. This raises the question of whether irradiation with an AC magnetic field with $f$ of $500 \mathrm{kHz}$ and $H_{\mathrm{ac}}$ of $37.3 \mathrm{kA} / \mathrm{m}$ for core-shell structured nanoparticles with $d$ of $15 \mathrm{~nm}$ and $K$ of $1.7 \times 10^{4} \mathrm{~J} / \mathrm{m}^{3}$ are really the optimum conditions. However, it is difficult to apply the other guiding principle to maximize hysteresis loss of ferromagnetic nanoparticles because thermally assisted reversals of $\boldsymbol{\mu}$ occur stochastically before the barrier disappears at $H_{K}$. Recalling that the characteristic time of thermal fluctuation was estimated to be a few hundred nanoseconds even in a zero magnetic field, the conditions used by Lee et al. are outside the scope of applicability of conventional models for ferromagnetic nanoparticles at a temperature of absolute zero and for superparamagnetic nanoparticles in a zero magnetic field. Consequently, new guiding principles to maximize heat dissipation $P_{H}$ are required. The second point is that $H_{\mathrm{ac}}=37.3 \mathrm{kA} / \mathrm{m}$ is much larger than the exposure restriction for this waveband [52]. This point is examined further in Section 5. The next section will present results of recent numerical studies on the behavior of nanoparticles under conditions outside the scope of applicability of conventional models. This knowledge will be useful to establish sophisticated guiding principles that are adapted to advanced technologies that control the size, shape, and composite structure of nanoparticles.

\section{Recent Numerical Simulations for Novel Responses to AC Magnetic Fields}

To further improve the guiding principles for the design of magnetic nanoparticles, we must clarify the behavior of nanoparticles under conditions outside the scope of applicability of conventional models. However, it is difficult to discuss nonlinear nonequilibrium responses algebraically; as an alternative, numerical simulation has been performed extensively because of recent advances in computing speed. Noteworthy results obtained from these studies will be introduced in this section. To fully discuss their features from the viewpoint of efficiency, the results are shown as the ratio of the simulated value of $P_{H}$ to the theoretical upper limit of $P_{H}: P_{H \text { Max }}$, where $P_{H \text { Max }}$ is expressed as $4 \mu_{0} M_{s} H_{\mathrm{ac}} \cdot f \cdot \rho^{-1}$ for irradiating AC magnetic field $H_{\mathrm{ac}} \sin (2 \pi f \cdot t)$, because the loss dissipated in one cycle is the area of the hysteresis loop.

In most of the simulations, it was assumed that magnetic nanoparticles were individually delivered to tumor tissues and accumulate randomly inside them, apart from the present status of this treatment [53]. Because the actual concentration of nanoparticles in tumors $c$ does not exceed $10 \mathrm{~kg} / \mathrm{m}^{3}\left(10 \mathrm{mg} / \mathrm{cm}^{3}\right)$ as stated above, effects caused by dipole-dipole interactions $J_{\mathrm{dd}}$ between the accumulated nanoparticles were considered insignificant at room temperature. For example, at the mean distance $\langle r\rangle \approx d$. $\rho^{1 / 3} \cdot c^{-1 / 3}, J_{\mathrm{dd}} / k_{B} \approx \mu_{0} \mu^{2} /\left(\langle r\rangle^{3} k_{B}\right)$ is estimated to be $25 \mathrm{~K}$ for magnetite nanoparticles with $d=15 \mathrm{~nm}, M_{s}=$ $450 \mathrm{kA} / \mathrm{m}$, and $c=10 \mathrm{~kg} / \mathrm{m}^{3}$. Thus, the nanoparticles in this hyperthermia treatment simulation were considered magnetically isolated from each other.
4.1. Néel Relaxation in Magnetic Fields. In a magnetically isolated nanoparticle, the potential energy, $U$, with respect to the direction of $\boldsymbol{\mu}$ is simply given by the sum of magnetic anisotropy energy and Zeeman energy. As a first approximation, uniaxial magnetic anisotropy has usually been assumed for the former term, although it contains contributions from various kinds of magnetic anisotropy such as shape, crystalline, and surface anisotropy. In this case, $U$ can be expressed as

$$
U(\phi, \psi)=K V \sin ^{2} \phi-\mu_{0} \mu H_{\mathrm{ac}} \sin (2 \pi f \cdot t) \cos \psi,
$$

where $\phi$ is the angle between the easy axis and $\boldsymbol{\mu}$, and $\psi$ is the angle between $\boldsymbol{\mu}$ and $\mathbf{H}$. The detailed trajectories of $\boldsymbol{\mu}$ in this potential can be precisely simulated by solving the stochastic Landau-Lifshitz-Gilbert equations [53-57]. However, we are only interested in the reversal of $\boldsymbol{\mu}$ once every microsecond because the frequency used for hyperthermia treatment is limited. Carrey et al. calculated the behavior of $\boldsymbol{\mu}$ using a well-known coarse-grained approach or "two-level approximation" $[58,59]$, which considers thermally activated reversals between the metastable directions via the midway saddle point in the energy barrier. In this calculation, easy axes of the accumulated nanoparticles were assumed to be fixed. This assumption seems valid when the nanoparticles are strongly anchored to structures resembling organelles.

Figures 4(a), 5(a), and 6(a) show contour plots of $P_{H} /$ $P_{H \text { Max }}$ calculated for cobalt ferrite, manganese ferrite and their core-shell nanoparticles introduced above, respectively, where the time evolution of the occupation probabilities of the directions parallel to the randomly oriented easy axes are simulated in the same way as Carrey et al. using the parameters given in Table 1 . At low $H_{\mathrm{ac}}$ of $1 \mathrm{kA} / \mathrm{m}$, $P_{H} / P_{H \text { Max }}$ of the core-shell nanoparticles increases with $f$, and a single maximum is found at a peak frequency, $f_{p}$, of $110 \mathrm{kHz}$ (Figure 6(a)). This behavior is consistent with the above prediction that $P_{H}$ is maximized when $f^{-1}$ is adjusted to the Néel relaxation time. It is notable that $f_{p}$ shifts to higher frequency as $H_{\mathrm{ac}}$ increases. This acceleration of Néel relaxation can be attributed to lowering of the energy barrier by the Zeeman energy. As indicated by the dashed line in Figure 6(a), the shift of $f_{p}$ can be explained by $\tau_{N}\left(H_{\mathrm{ac}}\right)$ calculated using the conventional Brown's equation as follows [60]:

$$
\begin{aligned}
{\left[\tau_{N}\left(H_{\mathrm{ac}}\right)\right]^{-1}=} & f_{0} \cdot\left(1-h^{2}\right) \\
& \times\left\{(1+h) \exp \left[\left(\frac{-K V}{k_{B} T}\right)(1+h)^{2}\right]\right. \\
& \left.+(1-h) \exp \left[\left(\frac{-K V}{k_{B} T}\right)(1-h)^{2}\right]\right\},
\end{aligned}
$$

where $h$ is $H / H_{K}$. In Figure 6(a), $f_{p}$ at $H_{\mathrm{ac}}=20 \mathrm{kA} / \mathrm{m}$, a typical $H_{\mathrm{ac}}$ for hyperthermia treatment, is about 40 times faster than that in a zero magnetic field. This fact clearly indicates that maximum heat dissipation cannot be obtained if we prepare nanoparticles according to the conventional guiding principles expressed in (1)-(5). This problem becomes serious 


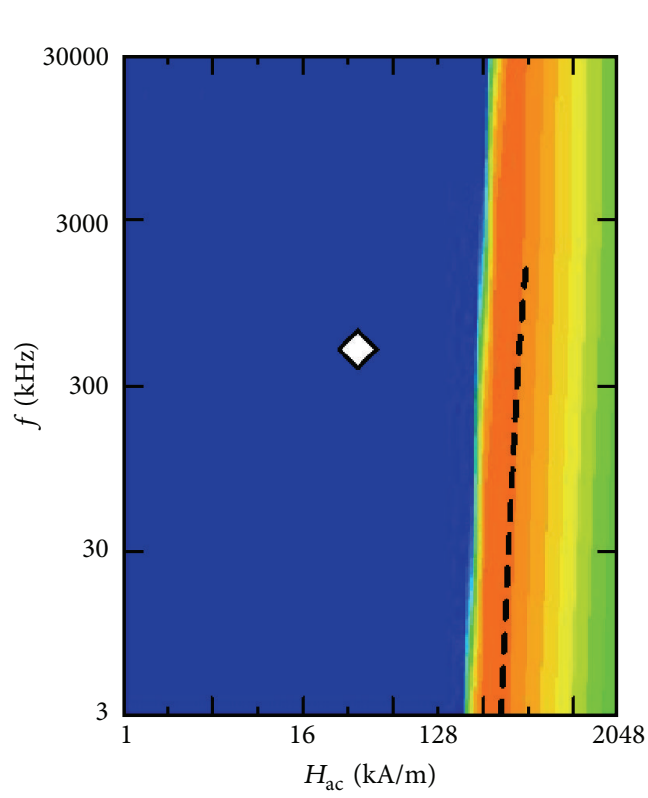

(a)

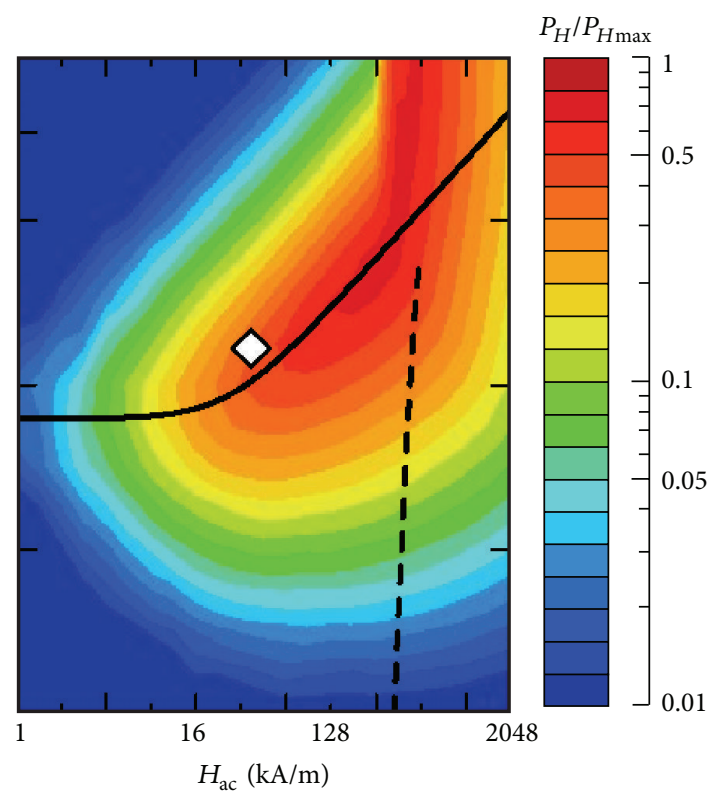

(b)

Figure 4: Calculated efficiency of heat dissipation by $\mathrm{CoFe}_{2} \mathrm{O}_{4}$ nanoparticles that are (a) nonrotatable and (b) rotatable. Dashed lines represent the Néel relaxation time $\left(2 \pi \tau_{N}\right)^{-1}$ and the solid line indicates $f_{p}$, which was calculated using (11). Diamonds denote the conditions used in the experiment.

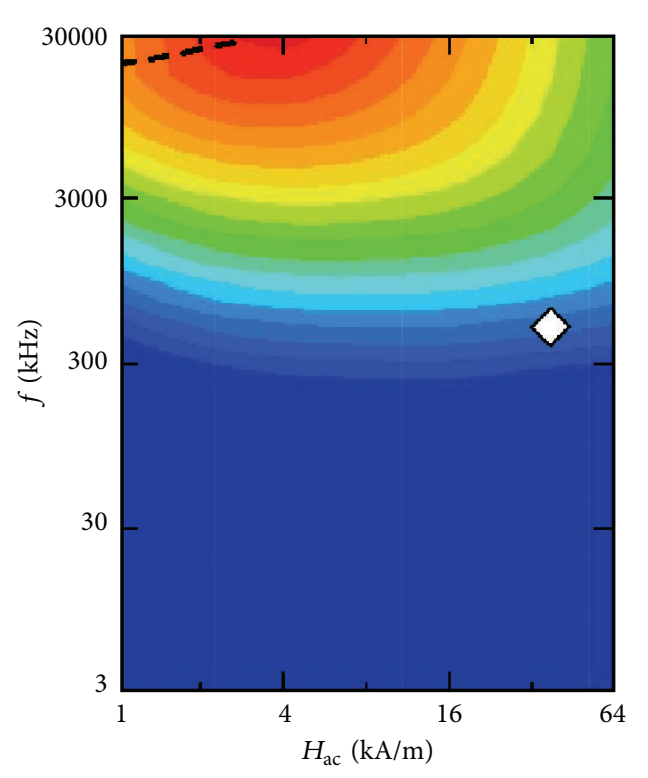

(a)

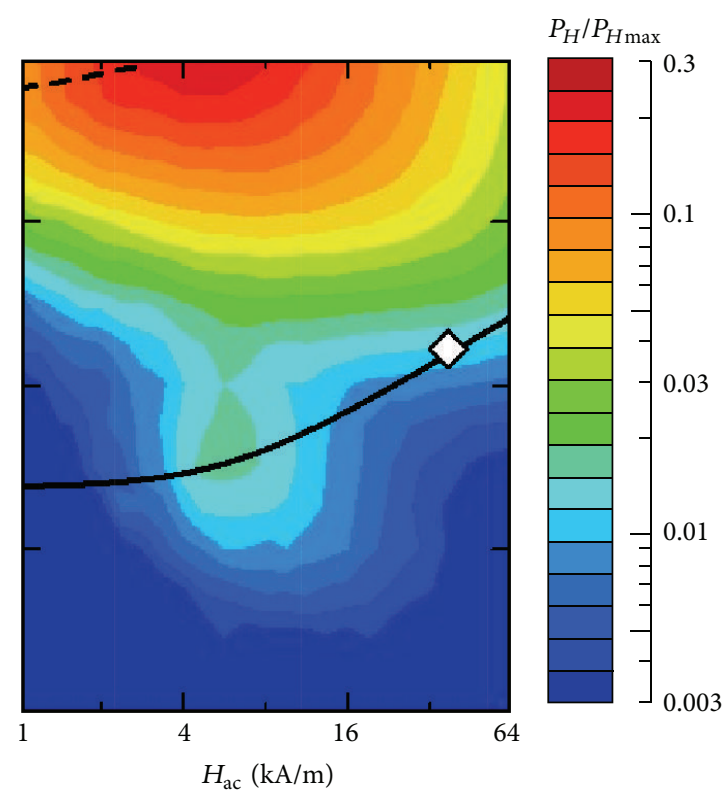

(b)

FIGURE 5: Calculated efficiency of heat dissipation by $\mathrm{MnFe}_{2} \mathrm{O}_{4}$ nanoparticles that are (a) non-rotatable and (b) rotatable. Dashed lines represent the Néel relaxation time $\left(2 \pi \tau_{N}\right)^{-1}$ and the solid line indicates $f_{p}$, which was calculated using (11). Diamonds denote the conditions used in the experiment.

when monodisperse nanoparticles are synthesized, although we barely noticed the problem because we used polydisperse nanoparticles with a broad distribution of $\tau_{N}$.

It is very important that these calculated results are compared with experimental data, even under only one set of conditions with $f=500 \mathrm{kHz}$ and $H_{\mathrm{ac}}=37.3 \mathrm{kA} / \mathrm{m}$.
In Figure 6(a), $40 \%$ of $P_{H \text { Max }}$, that is $1.4 \mathrm{MW} / \mathrm{kg}$, is expected for the core-shell nanoparticles at $f=500 \mathrm{kHz}$ and $H_{\mathrm{ac}}=37.3 \mathrm{kA} / \mathrm{m}$ (diamonds), whereas a larger value of 3.0 MW/kg was actually observed. In Figure 4(a), almost zero dissipation was calculated for the cobalt ferrite nanoparticles under the same conditions, because these nanoparticles 


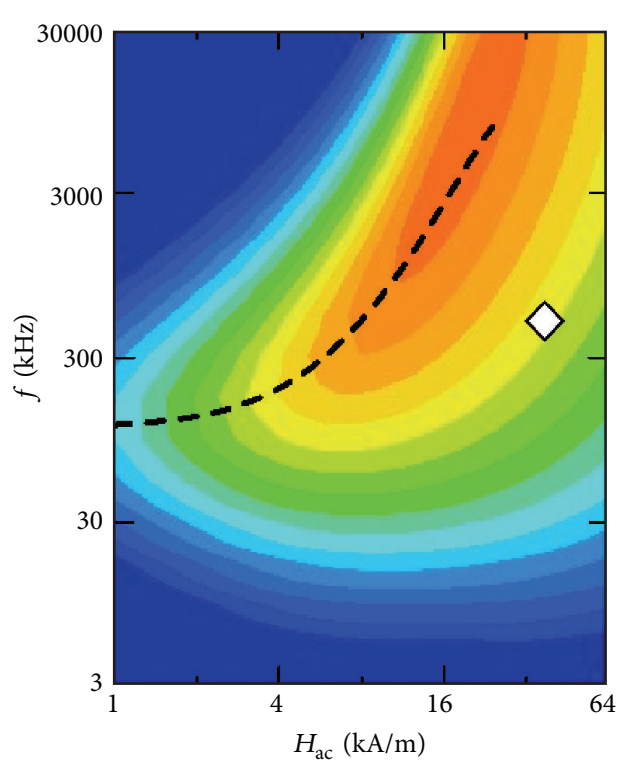

(a)

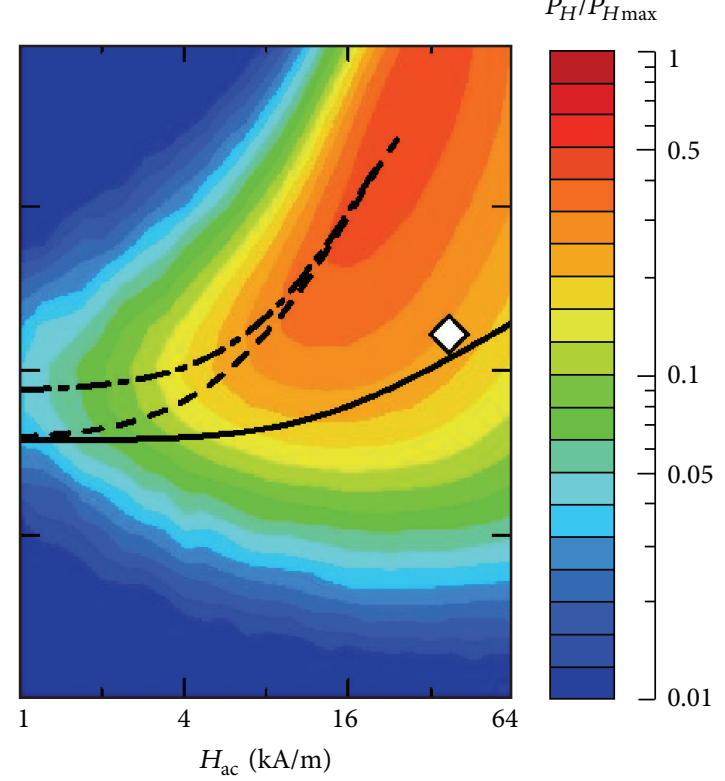

(b)

FIGURE 6: Calculated efficiency of heat dissipation by core-shell nanoparticles that are (a) non-rotatable and (b) rotatable. Dashed lines represent the Néel relaxation time $\left(2 \pi \tau_{N}\right)^{-1}$, the solid line indicates $f_{p}$, which was calculated using (11), and the dashed-dotted line shows the value calculated using (12). Diamonds denote the conditions used in the experiment.

are ferromagnetic, so no hysteresis loss is dissipated when $H_{\mathrm{ac}}=37.3 \mathrm{kA} / \mathrm{m}$ because it is sufficiently lower than $H_{K}=630 \mathrm{kA} / \mathrm{m}$. In contrast, considerable dissipation of $0.4 \mathrm{MW} / \mathrm{kg}$ was experimentally reported for the cobalt ferrite nanoparticles. In Figure 5(a), a small amount of dissipation is expected for the manganese ferrite nanoparticles under the same conditions, because these nanoparticles are typically superparamagnetic and little relaxation loss dissipates at $f=500 \mathrm{kHz}$ that is sufficiently lower than $\left[2 \pi \tau_{N}\left(H_{\mathrm{ac}}\right)\right]^{-1}$ of several tens of megahertz. However, a considerable dissipation of $0.2 \mathrm{MW} / \mathrm{kg}$ was experimentally reported for the manganese ferrite nanoparticles. Some of these inconsistencies may be attributed to the fact that the magnetic nanoparticles were easily rotatable in a low viscous liquid of toluene. Hence, Brownian rotations would be described next.

4.2. Brownian Relaxation in Magnetic Fields. In this subsection, ferromagnetic nanoparticles in Newtonian fluids are considered because toluene is a typical Newtonian fluid $(\eta=0.55 \mathrm{mPa} \cdot \mathrm{s})$, although the actual microviscoelasticity of the local environment in cancer cells is still unknown. In this case, the inertia of nanoparticles with a typical size of $10 \mathrm{~nm}$ can be neglected in considering their rotation by Brownian dynamics simulation $[61,62]$. In the inertia-less limit, frictional torque for the rotation of a sphere balances with magnetic torque $\mu(t) \times H(t)$ and Brownian torque $\lambda(t)$ as follows:

$$
\begin{gathered}
6 \eta V_{H} \cdot \boldsymbol{\omega}(t)=\mu_{0} \boldsymbol{\mu}(t) \times \mathbf{H}(t)+\lambda(t) \\
\left\langle\lambda_{i}(t)\right\rangle=0,
\end{gathered}
$$

$$
\left\langle\lambda_{i}\left(t_{1}\right) \lambda_{i}\left(t_{2}\right)\right\rangle=2 k_{B} T \cdot\left(6 \eta V_{H}\right) \cdot \delta\left(t_{1}-t_{2}\right),
$$

where $\boldsymbol{\omega}(t)$ is the angular velocity of rotation for the unit vector $\mathbf{e}(t)$ along the easy axis given by $d e / d t=\boldsymbol{\omega}(t) \times \mathbf{e}(t)$, and $\delta\left(t_{1}-t_{2}\right)$ is the Dirac delta function. Yoshida and Enpuku [63] simulated the rotation of ferromagnetic nanoparticles using the Fokker-Planck equation equivalent to the above relationships; they assumed that $\boldsymbol{\mu}(t)$ was permanently fixed at the direction parallel to $\mathbf{e}(t)$ as long as $H_{\mathrm{ac}}<H_{K}$. As a result, they confirmed that, at zero magnetic field limit, the frequency-dependence of heat dissipation exhibits a single maximum at $f_{p}=\left(2 \pi \tau_{B}\right)^{-1}$, as predicted by (2)-(5). They also found that $f_{p}$ increases with $H_{\mathrm{ac}}$ according to the equation:

$$
\begin{aligned}
2 \pi f_{p} & \approx \tau_{B}^{-1}\left[1+0.07\left(\frac{\mu_{0} \mu H_{\mathrm{ac}}}{k_{B} T}\right)^{2}\right]^{0.5} \\
& \approx \begin{cases}\tau_{B}^{-1} & \text { at } \mu_{0} \mu H_{\mathrm{ac}} \ll k_{B} T \\
0.5\left(\frac{\mu_{0} \mu H_{\mathrm{ac}}}{6 \eta V_{H}}\right) & \text { at } \mu_{0} \mu H_{\mathrm{ac}} \gg k_{B} T .\end{cases}
\end{aligned}
$$

This equation indicates that the driving force of the rotation changes from Brownian random torque to magnetic torque as $H_{\mathrm{ac}}$ increases.

As an example, this equation is applied to the cobalt ferrite nanoparticles discussed above. The solid curve in Figure 4(b) shows the values of $2 \pi f_{p}$ calculated using (11) with the parameters in Table 1 . The obtained line is close to the position of the diamond located at $f=500 \mathrm{kHz}$ and $H_{\mathrm{ac}}=37.3 \mathrm{kA} / \mathrm{m}$. In other words, the magnetic torque from the magnetic field at $37.3 \mathrm{kA} / \mathrm{m}$ happened to satisfy the conditions of rotating the cobalt ferrite nanoparticles 
with an appropriate delay to the alternation at $500 \mathrm{kHz}$; consequently, a considerable amount of heat, $3.7 \mathrm{MW} / \mathrm{kg}$, dissipates. Apart from the magnitude, this is the reason why $P_{H}=0.4 \mathrm{MW} / \mathrm{kg}$ was experimentally observed for the cobalt ferrite nanoparticles, despite the conventional prediction of no hysteresis loss under the experimental conditions. As exemplified here, delayed rotations are caused by magnetic torque (not Brownian torque) even at $H_{\mathrm{ac}}$ much lower than $H_{K}$, resulting in significant heat dissipation.

Researchers are also interested in the magnetic response when $H_{\mathrm{ac}}$ becomes comparable to $H_{K}$. In this case, the abovementioned assumption that $\boldsymbol{\mu}(t)$ is permanently fixed at the direction parallel to $\mathbf{e}(t)$ is invalid, because $\boldsymbol{\mu}(t)$ is canted from the easy axis by the Zeeman energy. Furthermore, $\boldsymbol{\mu}(t)$ stochastically reverses by thermal fluctuations even in ferromagnetic nanoparticles, because the Zeeman energy lowers the barrier height sufficiently. Therefore, I simultaneously computed the rotations of the nanoparticles using (8)-(10) with the thermally activated reversals of $\boldsymbol{\mu}(t)$ on the potential given by (6) [64]. Note that (8) is valid within the two-level approximation [65]. The results calculated for these cobalt ferrite nanoparticles are shown as the contour lines (and color difference) in Figure 4(b). Firstly, we are certain that, at $H_{\mathrm{ac}} \ll H_{K} \approx 630 \mathrm{kA} / \mathrm{m}$, the location of the ridge in the contour plot of $P_{H} / P_{H \text { Max }}$ is consistent with the solid line given by (11). This result indicates that ferromagnetic nanoparticles are rotated by the magnetic torque before the reversal of $\boldsymbol{\mu}(t)$ occurs within it. However, the ridge seems turn to the position extrapolated from the dashed curve given by (7) when $H_{\mathrm{ac}}$ becomes comparable to $H_{K}$. In other words, $\boldsymbol{\mu}(t)$ is promptly reversed before the rotation because the Néel relaxation is accelerated enough in this $H_{\mathrm{ac}}$ range. These relationships can be written as

$$
2 \pi f_{p} \approx\left[\tau_{N}\left(H_{\mathrm{ac}}\right)\right]^{-1}+\tau_{B}^{-1}\left[1+0.07\left(\frac{\mu_{0} \mu H_{\mathrm{ac}}}{k_{B} T}\right)^{2}\right]^{0.5} .
$$

This equation is an extended relationship of $\tau^{-1}=\tau_{N}^{-1}+\tau_{B}^{-1}$ ((3)) for a large AC magnetic field. It is noteworthy that the first term $\tau_{N}\left(H_{\mathrm{ac}}\right)$ usually becomes extremely small for ferromagnetic nanoparticles at $H_{\mathrm{ac}} \approx H_{K}$ in an aligned case $(\mathbf{e} / / \mathbf{H})$ or at $H_{\mathrm{ac}} \approx H_{K} / 2$ in tilted cases, while the second term is approximately expressed as $0.5\left(\mu_{0} \mu H_{\mathrm{ac}} / 6 \eta V_{H}\right)$ when $\mu_{0} \mu H_{\mathrm{ac}} \gg k_{B} T$. Therefore, the changeover from rotation to reversal occurs at $2 \pi f \approx 0.5\left(\mu_{0} \mu H_{K} / 6 \eta V_{H}\right)=K V /\left(6 \eta V_{H}\right)$ or $K V /\left(12 \eta V_{H}\right)$ for aligned and tilted cases, respectively. For example, this changeover frequency corresponds to $4 \mathrm{MHz}$ for the aligned cobalt ferrite nanoparticles with $d=12 \mathrm{~nm}$, $V / V_{H}=0.63, K=200 \mathrm{~kJ} / \mathrm{m}^{3}$, and $\eta=0.55 \mathrm{mPa} \cdot \mathrm{s}$. Importantly, the changeover frequency is independent of the size of nanoparticles as long as the ratio $V / V_{H}$ is constant. In other words, rotations predominate over the magnetic response at $1 \mathrm{MHz}$ even for much larger cobalt ferrite nanoparticles $\left(d=120 \mathrm{~nm},\left(2 \pi \tau_{B}\right)^{-1}=200 \mathrm{~Hz}\right)$. We must keep in mind that, even when ferromagnetic nanoparticles are large enough for their Brownian relaxation to be negligible, magnetic torque can easily rotate such nanoparticles at a time scale of microseconds if they are in a liquid phase. This knowledge is helpful when considering the optimal frequency for hyperthermia treatment, even if it is for a simplified system.

4.3. Easy Axes Oriented to the Directions Parallel, Perpendicular, or Oblique to the AC Field. As described above, the fast reversals of $\boldsymbol{\mu}(t)$ are predominant in the magnetic response of ferromagnetic nanoparticles at frequencies higher than the changeover frequency. The simulations, however, revealed that, at the frequencies, the rotation induces various kinds of stationary orientations of the easy axes $\mathbf{e}(t)$, which critically affect the reversals $[64,66]$. In this section, we also examine the results determined for cobalt ferrite nanoparticles with $d=12 \mathrm{~nm}, V / V_{H}=0.63, K=200 \mathrm{~kJ} / \mathrm{m}^{3}$, and $\eta=$ $0.55 \mathrm{mPa} \cdot \mathrm{s}$. In the initial state before irradiation with the AC magnetic field, the easy axes are set to be randomly oriented in the fluid, as shown in Figure 7(a). Therefore, in the first cycle, the major hysteresis loop obtained at $H_{\mathrm{ac}}=640 \mathrm{kA} / \mathrm{m}>$ $H_{K}$ is consistent with the magnetization curve predicted by the Stoner-Wohlfarth model (see the inset). If the irradiation of the AC magnetic field at $H_{\mathrm{ac}}=640 \mathrm{kA} / \mathrm{m}$ is continued in the simulation, the easy axes gradually turn toward the direction parallel to $\mathbf{H}$. Note that, in the case where the easy axis is not parallel to $\mathbf{H}$, the direction of $\boldsymbol{\mu}$ is not completely parallel to $\mathbf{H}$ even though $\boldsymbol{\mu}$ is already reversed at $H \geq H_{K}$. Therefore, a large magnetic torque proportional to $\sin \psi$ can turn the easy axis even if the magnetization seems almost saturated at $H \approx H_{K}$. For example, sin $\psi$ is 0.43 when cos $\psi$ is 0.9 . Consequently, a longitudinally oriented structure of the easy axes is formed in the fluid (see Figure $7(d)$ ). The formation of this nonequilibrium structure makes the dynamic hysteresis loop squarer than the initial curve, as shown in the inset of upper panel of Figure $7(d)$.

In contrast, the magnetization curve at $H_{\mathrm{ac}}=300 \mathrm{kA} / \mathrm{m}<$ $H_{K} / 2$ is a minor hysteresis loop, as shown in Figure 7(b). In this case, the easy axis turns toward the direction perpendicular to $\mathbf{H}$ and they maintain planar orientations if the ferromagnetic nanoparticles are continuously irradiated by an AC magnetic field at $H_{\mathrm{ac}}=300 \mathrm{kA} / \mathrm{m}$. A question now arises because we know that the longitudinal orientation is preferred when the Zeeman energy is considered. To clarify the reason for this, we consider an initial state in which a nanoparticle with an easy axis at angle $\theta$ has a magnetic moment $\boldsymbol{\mu}$ at a parallel direction $\psi=\theta$. When a small magnetic field $H<H_{K} / 2$ is applied to the nanoparticle, $\boldsymbol{\mu}$ immediately tilts to $\psi=\theta-\phi$ without reversals (see Figure $7(\mathrm{e})$ ), because the position of the local minimum on $U(\phi, \psi)$ is changed. Then, the magnetic torque, $-\mu_{0} \mu H \sin (\theta-$ $\phi)$, rotates $\boldsymbol{\mu}$ toward the longitudinal direction: $\psi \rightarrow 0$. Because $\boldsymbol{\mu}$ drags the easy axis, $\theta$ also decreases. In other words, the easy axis turns toward the direction parallel to $\mathbf{H}$. If $\mathbf{H}$ is reversed subsequently, the direction of $\boldsymbol{\mu}$ at this moment is almost antiparallel to $\mathbf{H}$ at $\psi=\theta+\pi-\phi$. Then $\psi$ instantly changes to $\theta+\pi+\phi$ because of the effect of variation of the minimum on $U(\phi, \psi)$ (see Figure $7(\mathrm{e})$ ). The magnetic torque at this stage, $-\mu_{0} \mu H \sin (\theta+\pi+\phi)=\mu_{0} \mu H \sin (\theta+\phi)$, forces $\boldsymbol{\mu}$ to rotate toward the direction $\psi=2 \pi$ via $\psi=(3 / 2) \pi$. Because $\boldsymbol{\mu}$ is bound on the easy axis, $\theta$ also increases. In other words, 

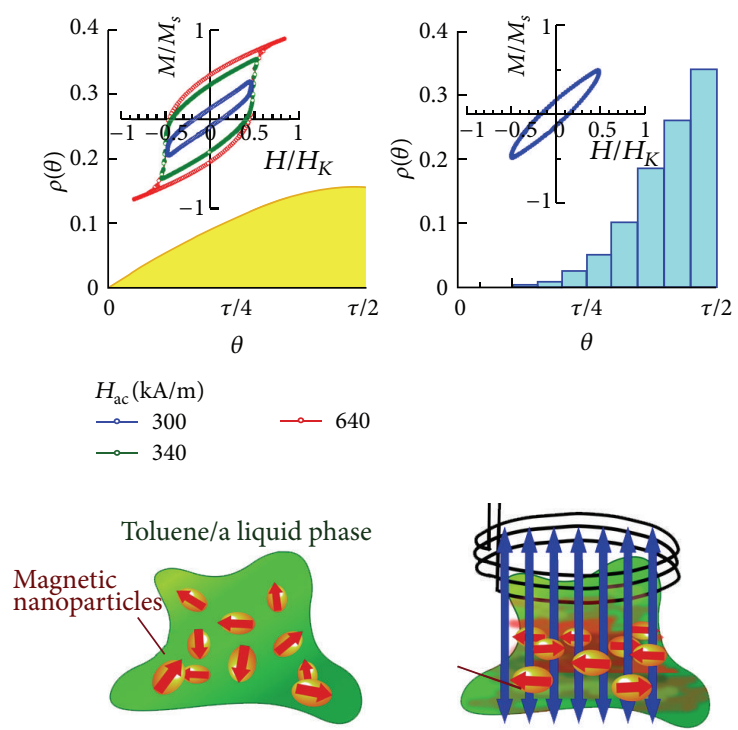

(a) $H_{\mathrm{ac}}=0$

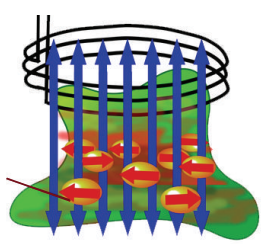

(b) $H_{\mathrm{ac}}=300 \mathrm{kA} / \mathrm{m}$
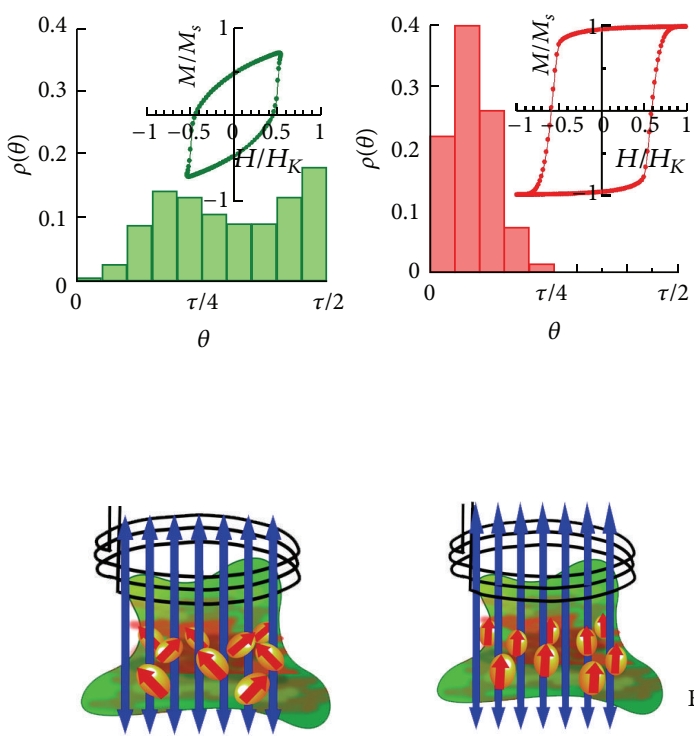

(c) $H_{\mathrm{ac}}=340 \mathrm{kA} / \mathrm{m}$

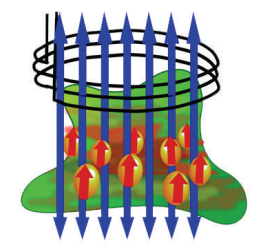

(d) $H_{\mathrm{ac}}=640 \mathrm{kA} / \mathrm{m}$

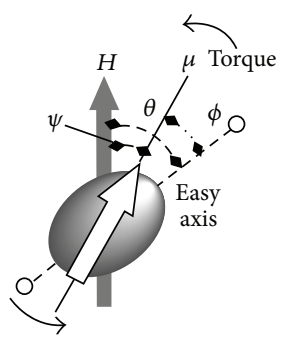

(e)

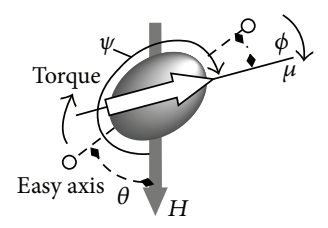

(f)

Figure 7: Calculated orientation distribution of the easy axes $\rho(\theta)$ of $\mathrm{CoFe}_{2} \mathrm{O}_{4}$ nanoparticles in (a) thermal equilibrium at $H=0$, and (b)(d) nonequilibrium steady states under AC magnetic field at various $H_{\mathrm{ac}}$ and $f=30 \mathrm{MHz}$.The inset shows the dynamic hysteresis loops. Diagrams of the magnetic torques in the AC field are depicted in (e) and (f), where the ellipsoid in each figure shows a nanoparticle, and the broken line, open and closed arrows indicate the directions of the easy axis, magnetic moment of the particle, and the AC magnetic field, respectively. The nonequilibrium structures under the high-frequency AC magnetic field are illustrated in the sketches in the lower column.

the easy axis starts to turn toward the plane perpendicular to $\mathbf{H}$. If the direction of $\mathbf{H}$ alternates at a high frequency, a planar orientation of the easy axis is formed on average, because $\mu_{0} \mu H \sin (\theta+\phi)$ is larger than $\mu_{0} \mu H \sin (\theta-\phi)$. This reduces the remanence of the hysteresis loop. In contrast, a longitudinal orientation is formed in a large AC magnetic field $H \geq H_{K}$ as discussed above, because $\boldsymbol{\mu}$ is always reversed to the direction parallel to $\mathbf{H}$ immediately after $\mathbf{H}$ is reversed. Overall, $\theta$ decreases toward 0 when the reversal of $\boldsymbol{\mu}$ occurs with alternation of the direction of $\mathbf{H}$, whereas $\theta$ increases toward $\pi / 2$ without reversal of $\boldsymbol{\mu}$.

This feature leads to formation of novel nonequilibrium structures such as the obliquely oriented state found at an intermediate amplitude of $H_{\mathrm{ac}}=340 \mathrm{kA} / \mathrm{m}$. Without considering thermal fluctuations, the reversals should occur in the range of $\theta$ from $0.15 \pi$ to $0.35 \pi$ for Stoner-Wohlfarth nanoparticles with $H_{K}=630 \mathrm{kA} / \mathrm{m}$, while $\boldsymbol{\mu}$ never reverses in the other ranges. If this feature simply applies, $\theta$ should decrease with time in the range between $0.15 \pi$ and $0.35 \pi$, whereas it should increase both between 0 and $0.15 \pi$ and between $0.35 \pi$ and $\pi / 2$. These variations certainly lead to formation of a bimodal $\rho(\theta)$ with double maxima at $\theta=0.15 \pi$ and $\pi / 2$, as found in Figure $7(\mathrm{c})$. Consequently, the easy axes are oriented in both the planes perpendicular and oblique to the magnetic field.

Concisely, in ferromagnetic nanoparticles in toluene or an aqueous phase, longitudinal, conical, or planar orientations are formed, irrespective of the free energy, as nonequilibrium structures under a high-frequency AC magnetic field. As a result, the major hysteresis loop becomes squarer and the minor loop becomes narrower compared with the magnetization curve calculated for randomly oriented nanoparticles. These variations of the area of the loops cause the maximum of $P_{H} / P_{H \text { Max }}$ to shift towards higher $H_{\mathrm{ac}}$ from the optimal conditions predicted by the conventional models in Section 2. This kind of averaging of the oscillating rotations, discussed using the cobalt ferrite nanoparticles as an example, should always occur as long as the alternation of the magnetic field is much more frequent than the characteristic time of rotation, $0.5\left(\mu_{0} \mu H_{\mathrm{ac}} / 6 \eta V_{H}\right)$. For this reason, these nonequilibrium structures would form in the radio-waveband used for hyperthermia treatment if the amplitude is somewhat smaller $(\sim 10 \mathrm{kA} / \mathrm{m})$ or the viscosity is considerably higher $(\sim 10 \mathrm{mPa} \cdot \mathrm{s})$. Therefore, we must keep in mind the important effects of nonequilibrium structures on heat dissipation when establishing the optimal design of ferromagnetic nanoparticles for hyperthermia treatment.

4.4. Magnetic Hysteresis of Superparamagnetic States. Let us leave ferromagnetic nanoparticles and move on to superparamagnetic manganese ferrite nanoparticles, from which a considerable amount of heat dissipation, $0.2 \mathrm{MW} / \mathrm{kg}$, was experimentally reported at $f=500 \mathrm{kHz}$. The orientation of $\boldsymbol{\mu}$ on these nanoparticles is easily equilibrated in the magnetic potential expressed in (6) within the scale of the Néel relaxation time $\tau_{N}\left(H_{\mathrm{ac}}=0\right)$ of $1 \times 10^{-8} \mathrm{~s}$. Therefore, little relaxation loss is expected using the conventional model. For this reason, I wish to examine this inconsistency from the viewpoint of the effects of slow rotations on the fast reversals in superparamagnetic nanoparticles.

The contour lines (and color difference) in Figure 5(b) show the results obtained from the simultaneous simulation 


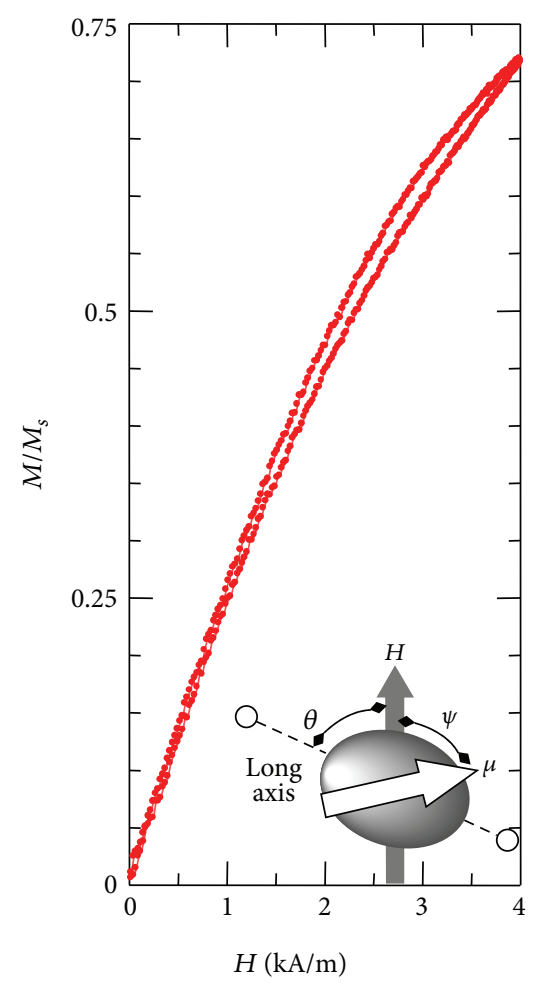

(a) $M-H$ curves

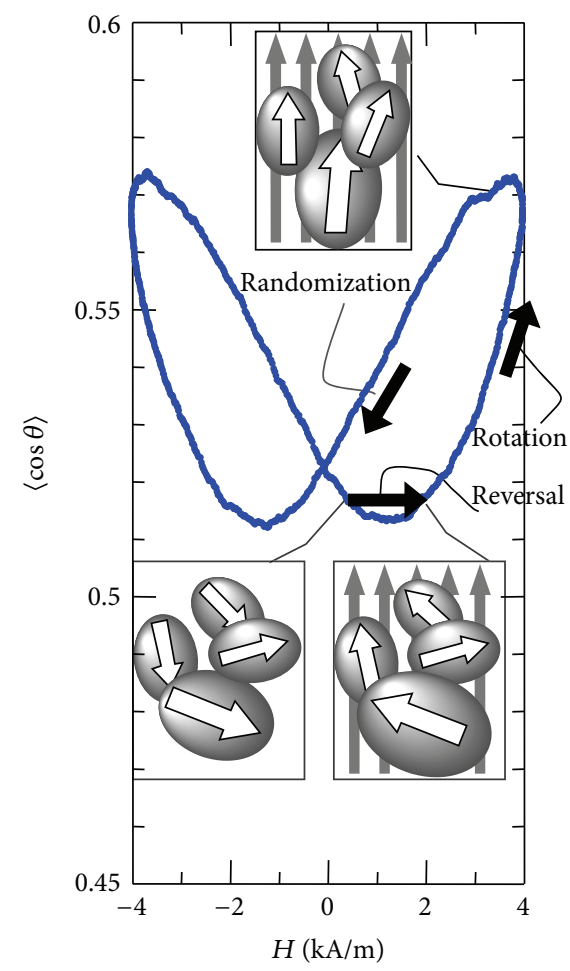

(b) Mean orientation

FIGURE 8: Calculated magnetic response of $\mathrm{MnFe}_{2} \mathrm{O}_{4}$ nanoparticles with an applied AC field with $H_{\mathrm{ac}}=4 \mathrm{kA} / \mathrm{m}$ and $f=100 \mathrm{kHz}$. (a) Steady magnetization curves, and (b) mean orientation of the easy axis of the nanoparticles, $\langle\cos \theta\rangle$. In the inset in (a), the ellipsoid shows a nanoparticle, and the broken line, open and closed arrows indicate the directions of the easy axis, magnetic moment of the particle, and the AC magnetic field, respectively. The variation of easy axis orientations is illustrated in the sketches in (b).

of rotations and reversals for the manganese ferrite nanoparticles. Firstly, we find a secondary maximum of $P_{H} / P_{H \text { Max }}$ around $f=100 \mathrm{kHz}$ in addition to the primary ridge of $P_{H} / P_{H \text { Max }}$ indicated by the dashed curve at frequencies of several tens of megahertz, which is explained by (7) for $\tau_{N}\left(H_{\mathrm{ac}}\right)$ above. To clarify the origin of the new kind of heat dissipation, the magnetization curve calculated under the conditions of $H_{\mathrm{ac}}=4 \mathrm{kA} / \mathrm{m}$ and $f=100 \mathrm{kHz}$ is presented in Figure 8(a). An S-shaped hysteresis loop without remanence is observed. In this cycle, the directions of the easy axes have butterfly-shaped hysteresis, as shown in Figure 8(b). This behavior is explained by the following atypical magnetic response in the period $f^{-1}(10 \mu \mathrm{s})$. Initially (at $t=0$ ), no magnetization exists because the occupation probabilities of $\boldsymbol{\mu}$ in the two stable directions parallel to the easy axis are equalized in a zero magnetic field. As $H$ increases, the occupation probability in the more stabilized direction immediately increases because of reversals on a time scale of $\tau_{N}$ ( $\leq 10 \mathrm{~ns}$ ). The reversed $\boldsymbol{\mu}$ in the stabilized direction is not completely parallel to $\mathbf{H}, \psi \neq 0$, and the magnetic torque $\mu_{0} \mu H \sin \psi$ turns the easy axis towards the direction of the field. The time constant of this process is approximately expressed as $\left[0.5\left(\mu_{0} \mu H_{\mathrm{ac}} / 6 \eta V_{H}\right)\right]^{-1}$ using the second term in (11). For the manganese ferrite nanoparticles, it is $3 \mu$ s when $H$ is $4 \mathrm{kA} / \mathrm{m}$. Therefore, rotation is not negligible in the peak period of the oscillations of $H$. Subsequently, $H$ decreases to zero at $t=0.5 f=5 \mu \mathrm{s}$, and the occupation probabilities are again equalized because reversal is rapid, so the magnetic torque disappears. Alternatively, the Brownian torque randomizes the orientation of the easy axis on a time scale of $\tau_{B}$ $(=2 \mu \mathrm{s})$. Therefore, competition between the magnetic and Brownian torques can cause the butterfly-shaped hysteresis of $\langle\cos \theta\rangle$. Because the equilibrium magnetization of the superparamagnetic nanoparticles with easy axes parallel to $H$ is higher than that of randomly oriented ones [58,67], the magnetization curve shows hysteresis without remanence. Consequently, a secondary maximum appears even though $\tau_{N} \ll \tau_{B}$ if the nanoparticles are rotatable. As discussed here, we should remove the stereotype of a single peak at a $2 \pi f_{p}$ value of $\tau^{-1}\left(=\tau_{N}^{-1}+\tau_{B}^{-1}\right)$.

Needless to say, there is still room for further study. For example, $P_{H}$ simulated at $f=500 \mathrm{kHz}$ and $H_{\mathrm{ac}}=37.3 \mathrm{kA} / \mathrm{m}$ is $0.13 \mathrm{MW} / \mathrm{kg}$, which is inconsistent with the observed $P_{H}$ of $0.2 \mathrm{MW} / \mathrm{kg}$. At present, it is unclear whether the difference can be attributed to the nontrivial polydisperse nature of the prepared sample or the accuracy of the simulations, because the experiment was performed under only one set of conditions with $f=500 \mathrm{kHz}$ and $H_{\mathrm{ac}}=37.3 \mathrm{kA} / \mathrm{m}$. Thus, measurement of $P_{H}$ under various conditions will be helpful to establish a model of heat dissipation in superparamagnetic nanoparticles. In addition, it is certain that the protocols of these simulations are also improvable, because it has been 
assumed that the direction of $\boldsymbol{\mu}$ is fixed at one of the local minima in the energy potential given by (6), although we know $\boldsymbol{\mu}$ stochastically explores all over the potential well [65]. Briefly, the magnetic torque is overestimated. Recently, more strict calculations were carried out and they also show the same kind of butterfly-shaped hysteresis [67]. As described here, much still remains to be done.

4.5. Intermediate State between Ferromagnetic and Superparamagnetic Nanoparticles. Core-shell nanoparticles, which can generate the largest amount of heat out of various nanoparticle structures, fit neither ferromagnetic $\left(\tau_{N}\left(H_{\mathrm{ac}}=0\right) \gg\right.$ $\left.f^{-1}\right)$ nor superparamagnetic $\left(\tau_{N}\left(H_{\mathrm{ac}}=0\right) \ll f^{-1}\right)$ conditions. This is because the value of the Néel relaxation time $\tau_{N}\left(H_{\mathrm{ac}}=0\right)$ calculated using the parameters in Table 1 is $1 \mu \mathrm{s}$, which is comparable with the alternation time of the AC magnetic field used in hyperthermia treatment. Furthermore, the Brownian relaxation time $\tau_{B}$ is also estimated to be $1 \mu \mathrm{s}$. Therefore, it is worth discussing this intermediate case before concluding this section. Figure 6(b) shows the results obtained by simultaneous simulation of rotation and reversal as contour lines (and color difference). In this figure, we are certain that location of the ridge in the contour plot of $P_{H} / P_{H \text { Max }}$ is consistent with neither the dashed curve (7) nor the solid curve (11), but instead with the dasheddotted curve given by (12). Furthermore, the iso-height contour lines, for example, the boundary between yellow and light green, shift toward lower frequency compared with the randomly fixed case in Figure 6(a). Figure 9 shows the magnetization curve and variation of the directions of the easy axes calculated for the core-shell nanoparticles under the conditions of $H_{\mathrm{ac}}=37.3 \mathrm{kA} / \mathrm{m}$ and $f=500 \mathrm{kHz}$. We observe eyeglass-shaped hysteresis in the variation of the directions of the easy axes. This behavior is attributed to complicated competition between normal rotations when $\boldsymbol{\mu}$ is parallel to $\mathbf{H}$, counter-rotations when $\boldsymbol{\mu}$ is antiparallel to $\mathbf{H}$, and randomization at $\mathbf{H} \approx 0$. The major point is that the first term seems to dominate the other terms, because the baseline of the eyeglass-shaped oscillations of the easy axes is considerably higher than the 0.5 expected for randomly oriented nanoparticles. This longitudinal orientation makes the dynamic hysteresis loop squarer and leads to an increase in $P_{H}$ (see Figure 9(a)). In addition to this effect, on average, oscillation of the directions of the easy axes induced by the alternation of the counter-rotations and randomization further increases $P_{H}$. Indeed, we can observe that the hysteresis loop of the rotatable nanoparticles in Figure 9(a) opens even in the higher magnetic field where the loop of the nonrotatable nanoparticles in Figure 9(a) is closed after all $\boldsymbol{\mu}$ are reversed. Overall, both the phenomena discussed for ferromagnetic and superparamagnetic nanoparticles contribute to amplification of the hysteresis loop area in this intermediate state; as a result, $P_{H}$ increases from $1.4 \mathrm{MW} / \mathrm{kg}$ for the nonrotatable case to $2.4 \mathrm{MW} / \mathrm{kg}$ for the rotatable one. We can say that this value is fairly consistent with the observed $P_{H}$ of $3 \mathrm{MW} / \mathrm{kg}$ in consideration that the simulation was carried out for completely isolated monodisperse nanoparticles with uniform uniaxial anisotropy.

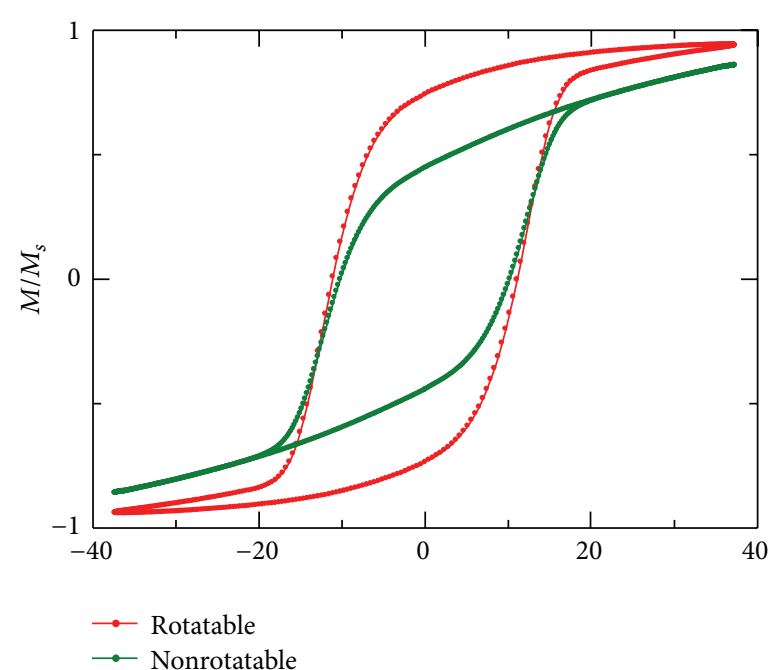

(a)

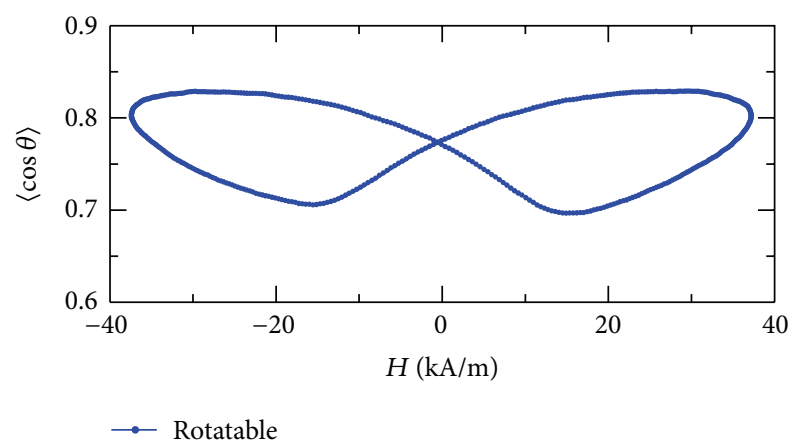

(b)

FIGURE 9: Calculated magnetic response of core-shell nanoparticles with an applied AC field with $H_{\mathrm{ac}}=37.3 \mathrm{kA} / \mathrm{m}$ and $f=500 \mathrm{kHz}$. (a) Steady magnetization curves, and (b) mean orientation of the easy axis of the nanoparticles, $\langle\cos \theta\rangle$.

\section{Optimized Design and Future Outlook}

Magnetic nanoparticles for thermotherapy, particularly rotatable nanoparticles, have been predicted to exhibit various novel responses to AC magnetic fields, as described above. Examples include magnetic hysteresis observed for superparamagnetic states and nonequilibrium structures with easy axes oriented to the directions parallel, perpendicular, or oblique to the magnetic field. These nonlinear and nonequilibrium phenomena cannot be explained using conventional models. Further systematic simulations and their experimental verification are required to establish sophisticated guiding principles for such magnetic nanoparticles. However, some feel that the heat generation of $3 \mathrm{MW} / \mathrm{kg}$ achieved by Lee et al. is sufficient for practical use in hyperthermia treatment, so more sophisticated guidelines may not be necessary. In this final section, we discuss this issue.

Tumors less than $0.01 \mathrm{~m}(=1 \mathrm{~cm})$ in size are considered difficult to find with existing diagnostic methods, so here we examine whether or not the heat dissipation from current magnetic nanoparticles is enough to treat hidden tumors of such size. According to Andrä et al. [68], raising 
the temperature of a tumor by $\Delta T$ requires heat generation of approximately $3 \lambda \Delta T R^{-2}$ without considering blood flow, where $\lambda$ is thermal conductivity and $2 R$ is the diameter of a tumor. If we assume $\lambda=0.6 \mathrm{WK}^{-1} \mathrm{~m}^{-1}, \Delta T=5 \mathrm{~K}$, and $2 R=0.005$ or $0.01 \mathrm{~m}$, the required heat generation would be 1.5 or $0.4 \mathrm{MW} / \mathrm{m}^{3}$, respectively. The rate of blood flow in tumor tissues is typically $1 \%$ per second by volume $(60 \mathrm{~mL} / \mathrm{min} / 100 \mathrm{~g})$ [69]; thus, when $\Delta T=5 \mathrm{~K}$, the heat transport caused by blood flow is estimated to be $0.2 \mathrm{MW} / \mathrm{m}^{3}$ using a value of $\sim 4 \mathrm{MJ}-\mathrm{m}^{-3} \mathrm{~K}^{-1}$ for the specific heat of blood. Therefore, the total cooling power of hidden tumors is between 0.6 and $2 \mathrm{MW} / \mathrm{m}^{3}$ for $\Delta T=5 \mathrm{~K}$. This assessment indicates that the amount of heat dissipation $P_{H}$ required to kill metastatic cancer cells is estimated to be within 0.3 and $1 \mathrm{MW} / \mathrm{kg}$ if we can expect a nanoparticle concentration within tumors of approximately $2 \mathrm{~kg} / \mathrm{m}^{3}$. The developed coreshell magnetic nanoparticles thus clearly enable adequate heat dissipation. However, are they actually suitable for use in hyperthermia treatment?

Note that Section 4 described how nanoparticles with $P_{H}$ of $3 \mathrm{MW} / \mathrm{kg}$ was obtained from irradiation using an $\mathrm{AC}$ magnetic field of $H_{\mathrm{ac}}=37.3 \mathrm{kA} / \mathrm{m}$ and $f=500 \mathrm{kHz}$. When this AC magnetic field is irradiated on a simple model body composed of a homogenous column with electrical conductivity $\sigma=0.2 \mathrm{Sm}^{-1}$ and radius $r=0.1 \mathrm{~m}$, the maximum voltage generated on the outer circumference is $V=\pi r^{2} 2 \pi f\left(\mu_{0} H_{\mathrm{ac}}\right)=4,600 \mathrm{~V}$ per revolution, at which point the eddy current loss $P_{e}=1 / 2 \pi^{2} \mu_{0}^{2} \sigma r^{2} f^{2} H_{\mathrm{ac}}^{2}$ is $5 \mathrm{MW} / \mathrm{m}^{3}$ $\left(5 \mathrm{~W} / \mathrm{cm}^{3}\right)$. This heat generation is sufficient to raise the temperature of thermally insulated tissues by $10 \mathrm{~K}$ or more in 10 seconds. For this reason, we cannot ignore the side effects of $P_{e}$ on normal tissues, although the model assuming a constant $\sigma$ is oversimplified. According to guidelines published by the International Commission on Non-Ionizing Radiation Protection [52], the upper limit for work-related exposure of the torso is $10 \mathrm{~W} / \mathrm{kg}$ (corresponding to $0.01 \mathrm{MW} / \mathrm{m}^{3}$ ). However, because this value is the upper limit for routine exposure, it may not be indicative of the maximum exposure in medical treatment. A slightly more specific value can be calculated as follows. Heat generation only occurs in the outer edge of a human body if a magnetic field is irradiated over the whole body; therefore, the heated region can be considered as a cylinder that is a few centimeters thick. This region can be cooled from the body surface area in medical treatment. Its cooling power, $\lambda \partial^{2} T / \partial r^{2} \sim \lambda \Delta T(\Delta r)^{-2}$, is roughly estimated to be $0.03 \mathrm{MW} / \mathrm{m}^{3}$ under the conditions of $\Delta T=$ $20 \mathrm{~K}$ and $\Delta r=0.02 \mathrm{~m}$. Because blood vessels expand when temperature rises, blood flow increases even in tissues with little blood flow normally. In subcutaneous tissues, for example, a blood flow rate of approximately $0.2 \%$ per second by volume $(12 \mathrm{~mL} / \mathrm{min} / 100 \mathrm{~g})$ has been reported at $42^{\circ} \mathrm{C}[69,70]$. Under these conditions, calculating the heat transport caused by blood flow using the same method yields a value of $0.03 \mathrm{MW} / \mathrm{m}^{3}$ when the temperature difference from the outside of the irradiated region is set at $\Delta T=4 \mathrm{~K}$. These values are the result of rough calculations that do not consider the detailed structure of a human body [71], but their sum, which is about $0.06 \mathrm{MW} / \mathrm{m}^{3}$, can be used as an approximation of cooling ability. When a magnetic field is applied to the model body mentioned above, this value corresponds to $P_{e}$ for the condition $H_{\mathrm{ac}} f=2 \times 10^{9} \mathrm{Am}^{-1} \mathrm{~s}^{-1}$. Calculating the behavior of the above-mentioned core-shell nanoparticles $(d=15 \mathrm{~nm}$ ) within this restriction (see Figure 10) shows that $P_{H}$ does not reach the requirement of $0.3 \mathrm{MW} / \mathrm{kg}$. However, Figure 10 indicates that if the size of the particle is increased slightly, sufficient $P_{H}$ can be obtained from the rotatable nanoparticles at higher $H_{\mathrm{ac}}$ (equivalent to lower $f$ ) even under this restriction, and adequate heating is expected inside hidden tumors with a diameter of $0.01 \mathrm{~m}$ without serious side effects on normal tissues from $P_{e}$.

Our discussion up to this point applies to treatment using continuous irradiation where heat balance holds. Irradiation time and interval can be controlled in medical treatment. For example, when tumors with a specific heat of $4 \mathrm{MJm}^{-3} \mathrm{~K}^{-1}$ containing the above-mentioned core-shell nanoparticles with a concentration $c$ of $2 \mathrm{~kg} / \mathrm{m}^{3}$ were irradiated with an AC magnetic field of $H_{\mathrm{ac}}=37.3 \mathrm{kA} / \mathrm{m}$ and $f=500 \mathrm{kHz}$, heat of approximately $c P_{H}=6 \mathrm{MW} / \mathrm{m}^{3}$ was generated. Relative to this value, the quantity of heat diffused to the surrounding areas from $10 \mathrm{~mm}$ tumors is negligible when $\Delta T<5 \mathrm{~K}$; thus, the temperature will rise by $5 \mathrm{~K}$ after approximately 3 seconds. Because the eddy current loss $P_{e}$ in this case is $5 \mathrm{MW} / \mathrm{m}^{3}$, it will take approximately 4 seconds for the temperature of normal tissue to rise by $5 \mathrm{~K}$. Stopping irradiation after 3 seconds will thus enable selective heating of tumors by $5 \mathrm{~K}$ or more. This is an extreme example; however, it does indicate that there is another option apart from continuous irradiation. The ratio of $c P_{H}$ to $P_{e}$ is important. Although obtaining robust values requires detailed protocol, a factor of 4-5 or so might be a criterion for $c P_{H} / P_{e}$. As an example, we calculated $c P_{H} / P_{e}$ for the core-shell nanoparticles and found that this condition is satisfied for lower frequencies/smaller amplitudes than those indicated by the solid line in Figure 11 [72]. This finding reflects the fact that $P_{H}$ is the area of the $M-H$ curve $\times$ frequency, which is proportional to $H_{\mathrm{ac}} f$ at most, whereas $P_{e}$ increases in proportion to $\left(H_{\mathrm{ac}} f\right)^{2}$, as previously described. Because it is impossible to attain a rise in temperature of $5 \mathrm{~K}$ if $c P_{H}$ is at least 0.6 (or 2) $\mathrm{MW} / \mathrm{m}^{3}$, irradiation must therefore be conducted using a higher frequency and larger amplitude to ensure that this condition is met (see dashed lines in Figure 12 [72]). Ultimately, stronger, faster conditions are needed to destroy cancer cells, and weaker, slower conditions are needed to limit damage to normal tissue. Using the core-shell nanoparticles of $d=15 \mathrm{~nm}$, a frequency of $f=500 \mathrm{kHz}$ is thus acceptable, but $H_{\mathrm{ac}}$ needs to be maintained at $9 \mathrm{kA} / \mathrm{m}$ to resolve the conflicting requirements.

As discussed above, the combination of the core-shell nanoparticles of $d=15 \mathrm{~nm}$ and $K=1.7 \times 10^{4} \mathrm{~J} / \mathrm{m}^{3}$ with an AC magnetic field of $f=500 \mathrm{kHz}$ and $H_{\mathrm{ac}}=37.3 \mathrm{kA} / \mathrm{m}$ may not be optimal. A narrow range of combinations of these parameters will facilitate efficient hyperthermia treatment and prevent side effects. We have not yet optimized the conditions for hyperthermia treatment; however, establishing the optimal combinations may be difficult, particularly if 
$f(\mathrm{kHz})$

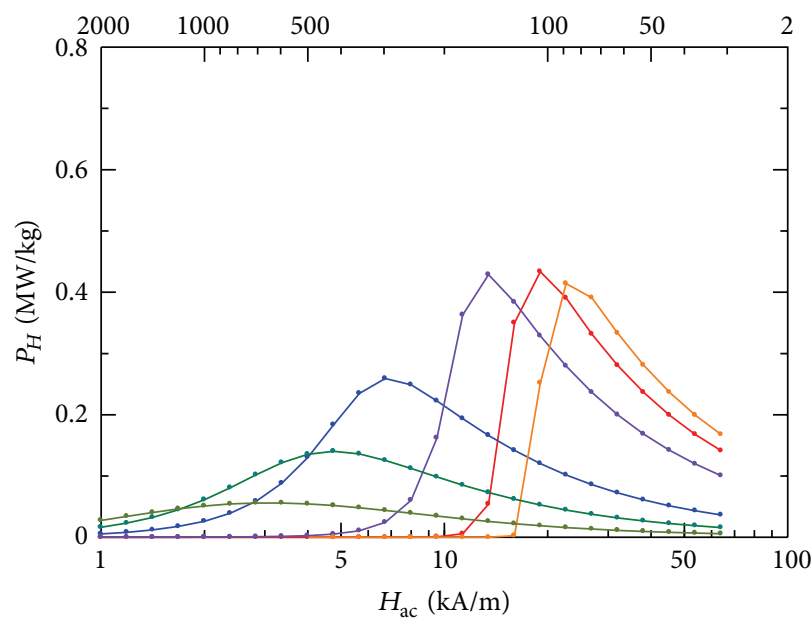

$\begin{array}{ll}d(\mathrm{~nm}) & \\ \rightarrow 13 & \rightarrow 18 \\ \rightarrow 14 & \rightarrow 22 \\ -15 & \rightarrow 28\end{array}$

(a) Nonrotatable

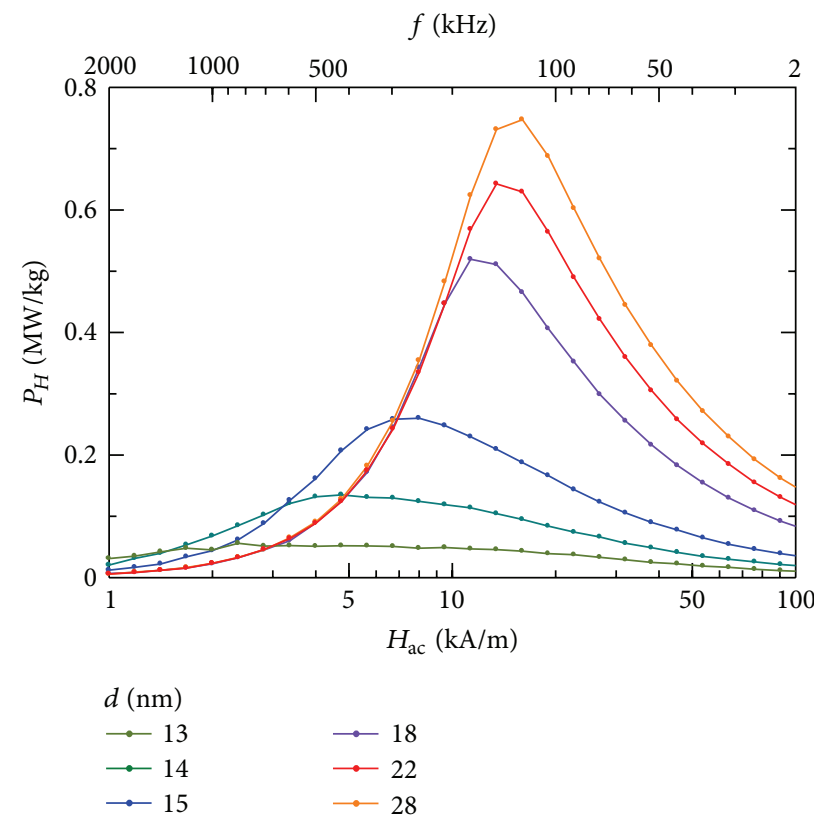

(b) Rotatable

FIGURE 10: Calculated heat dissipation by core-shell nanoparticles that are (a) non-rotatable and (b) rotatable, where $H_{\text {ac }} f$ is always $2 \times$ $10^{9} \mathrm{Am}^{-1} \mathrm{~s}^{-1}$ (corresponding to the restriction that the eddy current loss $P_{e}$ is $0.06 \mathrm{MW} / \mathrm{m}^{3}$ in normal tissue). The size $d$ is changed in the simulation, but the other parameters were fixed at the values shown in Table 1.
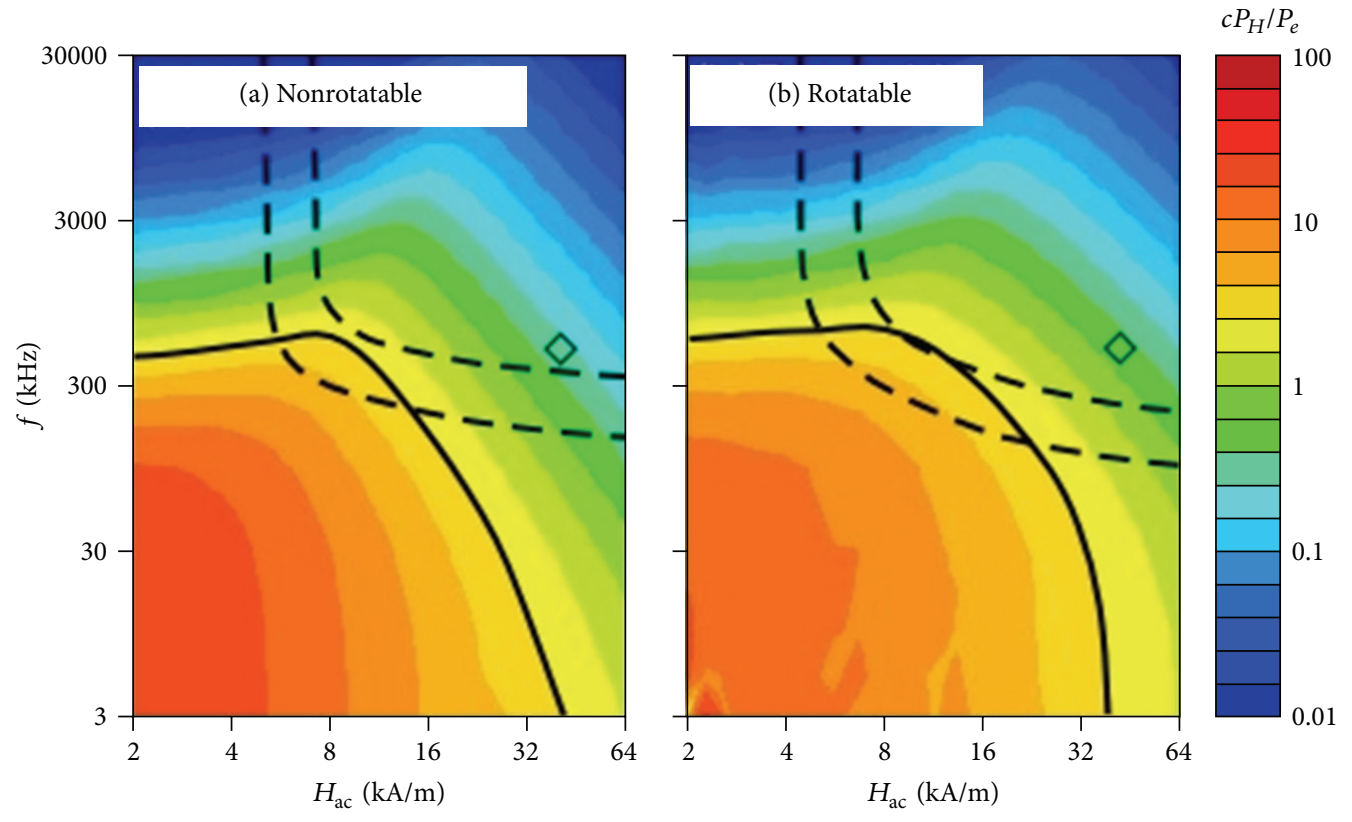

FIGURE 11: Calculated selection ratio $c P_{H} / P_{e}$ for core-shell nanoparticles in AC magnetic fields with various $H_{\text {ac }}$ and $f$. Rotatable nanoparticles are compared with randomly oriented ones. The dashed lines show the isoplethic curves at $P_{H}=0.3$ and $1 \mathrm{MW} / \mathrm{kg}$ (see Figure 12), while the solid lines show the isoplethic curves at $c P_{H} / P_{e}=4 .{ }^{*}$ Reproduced from Mamiya [72] with permission (Copyright 2012 TIC).

a trial and error approach is used. The routes used to synthesize magnetic nanoparticles of controlled size, shape, and composite structure have become increasingly advanced, as described in this paper. Dramatic advances in computing speed have also promoted numerical simulation of nonlinear nonequilibrium responses to AC magnetic fields. If we continue to improve material design on the bases of such advanced nanotechnology and computer simulations, optimal conditions will eventually be clarified. Remarkable advances are still continually being reported in clinical trials are being conducted, even though the combination of nanoparticles and oscillation of the equipment has not been 

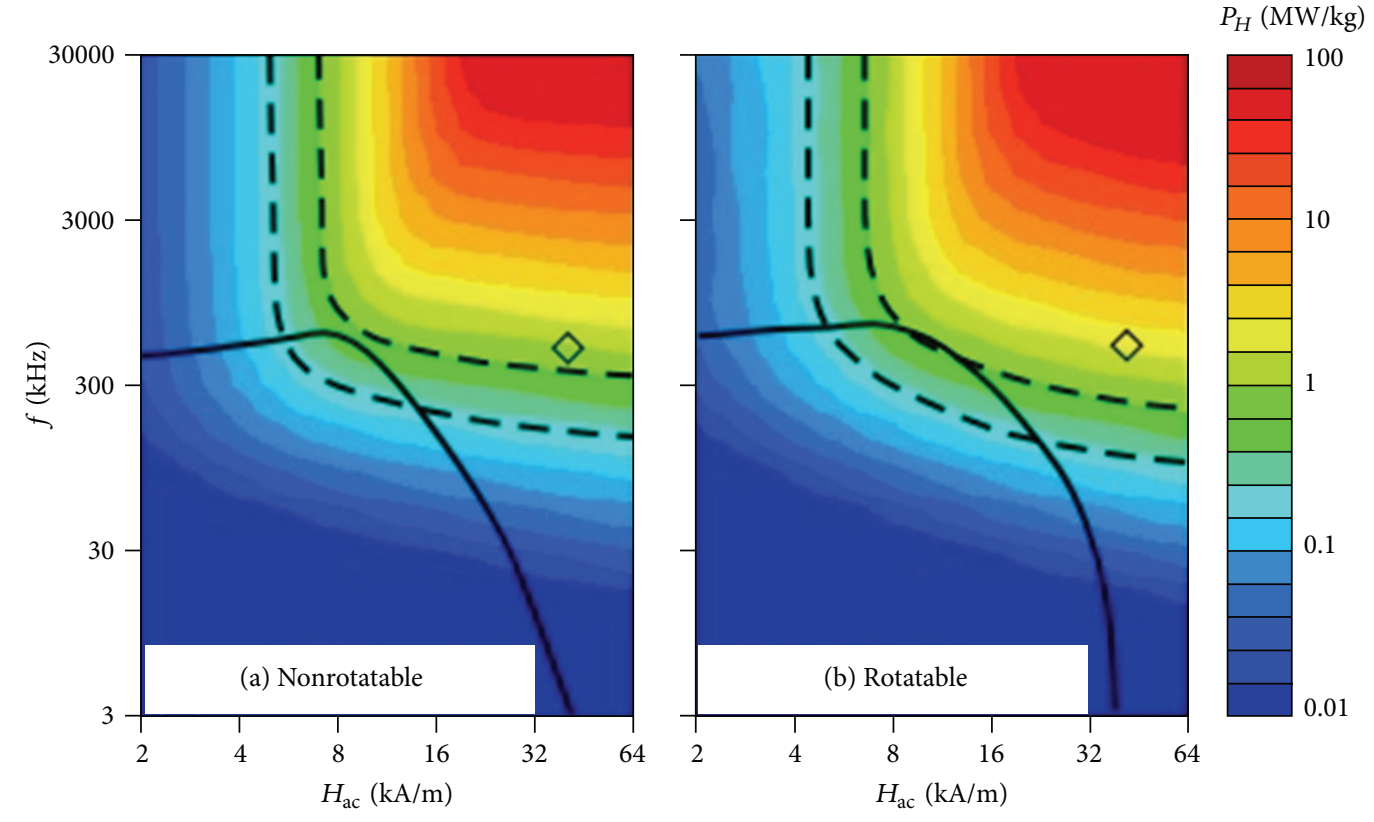

FIGURE 12: Calculated magnetic loss $P_{H}$ for core-shell nanoparticles in AC magnetic fields for various $H_{\mathrm{ac}}$ and $f$. Rotatable nanoparticles are compared with randomly oriented ones. The dashed lines show the isoplethic curves at $P_{H}=0.3$ and $1 \mathrm{MW} / \mathrm{kg}$, while the solid lines show the isoplethic curves at the selection ratio $c P_{H} / P_{e}=4$ (see Figure 11). ${ }^{*}$ Reproduced from Mamiya [72] with permission (Copyright 2012 TIC).

optimized yet [73, 74]. Once optimization improves local heating ability, then thermotherapy should be established as a fourth or fifth standard cancer treatment method to reduce the disease burden of a patient.

\section{Acknowledgment}

This work was partly supported by a Grant-in-Aid for Scientific Research (No. 24310071).

\section{References}

[1] W. C. Dewey, L. E. Hopwood, S. A. Sapareto, and L. E. Gerweck, "Cellular responses to combinations of hyperthermia and radiation," Radiology, vol. 123, no. 2, pp. 463-474, 1977.

[2] S. Mornet, S. Vasseur, F. Grasset, and E. Duguet, "Magnetic nanoparticle design for medical diagnosis and therapy," Journal of Materials Chemistry, vol. 14, no. 14, pp. 2161-2175, 2004.

[3] P. Tartaj, M. Del Puerto Morales, S. Veintemillas-Verdaguer, T. González-Carreño, and C. J. Serna, "The preparation of magnetic nanoparticles for applications in biomedicine," Journal of Physics D, vol. 36, no. 13, pp. R182-R197, 2003.

[4] T. Neuberger, B. Schöpf, H. Hofmann, M. Hofmann, and B. von Rechenberg, "Superparamagnetic nanoparticles for biomedical applications: possibilities and limitations of a new drug delivery system," Journal of Magnetism and Magnetic Materials, vol. 293, no. 1, pp. 483-496, 2005.

[5] Q. A. Pankhurst, N. K. T. Thanh, S. K. Jones, and J. Dobson, "Progress in applications of magnetic nanoparticles in biomedicine," Journal of Physics D, vol. 42, no. 22, Article ID 224001, 2009.
[6] K. M. Krishnan, "Biomedical nanomagnetics: a spin through possibilities in imaging, diagnostics, and therapy," IEEE Transactions on Magnetics, vol. 46, no. 7, pp. 2523-2558, 2010.

[7] B. Jeyadevan, "Present status and prospects of magnetite nanoparticles-based hyperthermia," Journal of the Ceramic Society of Japan, vol. 118, no. 1378, pp. 391-401, 2010.

[8] I. Sharifi, H. Shokrollahi, and S. Amiri, "Ferrite-based magnetic nanofluids used in hyperthermia applications," Journal of Magnetism and Magnetic Materials, vol. 324, no. 6, pp. 903-915, 2012.

[9] S. J. DeNardo, G. L. DeNardo, A. Natarajan et al., "Thermal dosimetry predictive of efficacy of111In-ChL6 nanoparticle AMF-induced thermoablative therapy for human breast cancer in mice," Journal of Nuclear Medicine, vol. 48, no. 3, pp. 437-444, 2007.

[10] P. Wust, U. Gneveckow, M. Johannsen et al., "Magnetic nanoparticles for interstitial thermotherapy-feasibility, tolerance and achieved temperatures," International Journal of Hyperthermia, vol. 22, no. 8, pp. 673-685, 2006.

[11] R. Hergt, R. Hiergeist, I. Hilger et al., "Maghemite nanoparticles with very high AC-losses for application in RF-magnetic hyperthermia," Journal of Magnetism and Magnetic Materials, vol. 270, no. 3, pp. 345-357, 2004.

[12] M. Ma, Y. Wu, J. Zhou, Y. Sun, Y. Zhang, and N. Gu, "Size dependence of specific power absorption of $\mathrm{Fe}_{3} \mathrm{O}_{4}$ particles in AC magnetic field," Journal of Magnetism and Magnetic Materials, vol. 268, no. 1-2, pp. 33-39, 2004.

[13] T. N. Brusentsova, N. A. Brusentsov, V. D. Kuznetsov, and V. N. Nikiforov, "Synthesis and investigation of magnetic properties of Gd-substituted Mn-Zn ferrite nanoparticles as a potential low-TC agent for magnetic fluid hyperthermia," Journal of Magnetism and Magnetic Materials, vol. 293, no. 1, pp. 298-302, 2005.

[14] G. Glöckl, R. Hergt, M. Zeisberger, S. Dutz, S. Nagel, and W. Weitschies, "The effect of field parameters, nanoparticle 
properties and immobilization on the specific heating power in magnetic particle hyperthermia," Journal of Physics, vol. 18, no. 38, pp. S2935-S2949, 2006.

[15] J. P. Fortin, C. Wilhelm, J. Servais, C. Ménager, J.-C. Bacri, and F. Gazeau, "Size-sorted anionic iron oxide nanomagnets as colloidal mediators for magnetic hyperthermia," Journal of the American Chemical Society, vol. 129, no. 9, pp. 2628-2635, 2007.

[16] G. Baldi, D. Bonacchi, C. Innocenti, G. Lorenzi, and C. Sangregorio, "Cobalt ferrite nanoparticles: the control of the particle size and surface state and their effects on magnetic properties," Journal of Magnetism and Magnetic Materials, vol. 311, no. 1, pp. 10-16, 2007.

[17] L. Y. Zhang, H.-C. Gu, and X.-M. Wang, "Magnetite ferrofluid with high specific absorption rate for application in hyperthermia," Journal of Magnetism and Magnetic Materials, vol. 311, no. 1, pp. 228-233, 2007.

[18] D.-H. Kim, D. E. Nikles, D. T. Johnson, and C. S. Brazel, "Heat generation of aqueously dispersed $\mathrm{CoFe}_{2} \mathrm{O}_{4}$ nanoparticles as heating agents for magnetically activated drug delivery and hyperthermia," Journal of Magnetism and Magnetic Materials, vol. 320, no. 19, pp. 2390-2396, 2008.

[19] J.-P. Fortin, F. Gazeau, and C. Wilhelm, "Intracellular heating of living cells through Néel relaxation of magnetic nanoparticles," European Biophysics Journal, vol. 37, no. 2, pp. 223-228, 2008.

[20] L.-M. Lacroix, R. B. Malaki, J. Carrey et al., "Magnetic hyperthermia in single-domain monodisperse FeCo nanoparticles: evidences for Stoner-Wohlfarth behavior and large losses," Journal of Applied Physics, vol. 105, no. 2, Article ID 023911, 4 pages, 2009.

[21] C. L. Dennis, A. J. Jackson, J. A. Borchers et al., "Nearly complete regression of tumors via collective behavior of magnetic nanoparticles in hyperthermia," Nanotechnology, vol. 20, no. 39, Article ID 395103, 2009.

[22] M. Gonzales-Weimuller, M. Zeisberger, and K. M. Krishnan, "Size-dependant heating rates of iron oxide nanoparticles for magnetic fluid hyperthermia," Journal of Magnetism and Magnetic Materials, vol. 321, no. 13, pp. 1947-1950, 2009.

[23] R. Sharma and C. J. Chen, "Newer nanoparticles in hyperthermia treatment and thermometry," Journal of Nanoparticle Research, vol. 11, no. 3, pp. 671-689, 2009.

[24] E. Kita, T. Oda, T. Kayano et al., "Ferromagnetic nanoparticles for magnetic hyperthermia and thermoablation therapy," Journal of Physics D, vol. 43, no. 47, Article ID 474011, 2010.

[25] B. Mehdaoui, A. Meffre, L.-M. Lacroix et al., "Large specific absorption rates in the magnetic hyperthermia properties of metallic iron nanocubes," Journal of Magnetism and Magnetic Materials, vol. 322, no. 19, pp. L49-L52, 2010.

[26] T. Kikuchi, R. Kasuya, S. Endo et al., "Preparation of magnetite aqueous dispersion for magnetic fluid hyperthermia," Journal of Magnetism and Magnetic Materials, vol. 323, no. 10, pp. 12161222, 2011.

[27] J.-H. Lee, J.-T. Jang, J.-S. Choi et al., "Exchange-coupled magnetic nanoparticles for efficient heat induction," Nature Nanotechnology, vol. 6, no. 7, pp. 418-422, 2011.

[28] S.-H. Noh, W. Na, J. Jang et al., "Nanoscale magnetism control via surface and exchange anisotropy for optimized ferrimagnetic hysteresis," Nano Letters, vol. 12, no. 7, pp. 3716-3721, 2012.

[29] K. Nakamura, K. Ueda, A. Tomitaka et al., "Self-heating temperature and $\mathrm{AC}$ hysteresis of magnetic iron oxide nanoparticles and their dependence on secondary particle size," IEEE Transactions on Magnetics, vol. 49, no. 1, pp. 240-243, 2013.
[30] C. Martinez-Boubeta, K. Simeonidis, A. Makridis et al., "Learning from nature to improve the heat generation of ironoxide nanoparticles for magnetic hyperthermia applications," Scientific Reports, vol. 3, article 1652, 2013.

[31] J. L. Dormann, D. Fiorani, and E. Tronc, "Magnetic relaxation in fine-particle systems," Advances in Chemical Physics, vol. 98, pp. 283-494, 1997.

[32] X. Batlle and A. Labarta, "Finite-size effects in fine particles: magnetic and transport properties," Journal of Physics D, vol. 35, no. 6, pp. R15-R42, 2002.

[33] H. Mamiya, Magnetic Properties of Nanoparticles, Yushodo, Tokyo, Japan, 2003.

[34] P. E. Jönsson, "Superparamagnetism and spin glass dynamics of interacting magnetic nanoparticle systems," Advances in Chemical Physics, vol. 128, pp. 191-248, 2004.

[35] P. C. Scholten, "How magnetic can a magnetic fluid be?" Journal of Magnetism and Magnetic Materials, vol. 39, no. 1-2, pp. 99106, 1983.

[36] H. Mamiya, I. Nakatani, and T. Furubayashi, "Blocking and freezing of magnetic moments for iron nitride fine particle systems," Physical Review Letters, vol. 80, no. 1, pp. 177-180, 1998.

[37] H. Mamiya, I. Nakatani, and T. Furubayashi, "Slow dynamics for spin-glass-like phase of a ferromagnetic fine particle system," Physical Review Letters, vol. 82, no. 21, pp. 4332-4335, 1999.

[38] H. Mamiya, I. Nakatani, and T. Furubayashi, "Phase transitions of iron-nitride magnetic fluids," Physical Review Letters, vol. 84, no. 26, pp. 6106-6109, 2000.

[39] A. Wiedenmann, M. Kammel, A. Heinemann, and U. Keiderling, "Nanostructures and ordering phenomena in ferrofluids investigated using polarized small angle neutron scattering," Journal of Physics, vol. 18, no. 38, pp. S2713-S2736, 2006.

[40] H. Kronmüller and M. Fähnle, Micromagnetism and the Microstructure of Ferromagnetic Solids, Cambridge University Press, Cambridge, UK, 2003.

[41] R. Hergt, S. Dutz, and M. Röder, "Effects of size distribution on hysteresis losses of magnetic nanoparticles for hyperthermia," Journal of Physics, vol. 20, no. 38, Article ID 385214, 2008.

[42] R. E. Rosensweig, "Heating magnetic fluid with alternating magnetic field," Journal of Magnetism and Magnetic Materials, vol. 252, pp. 370-374, 2002.

[43] S. S. Papell, US Patent No. 3, 215, 1965.

[44] T. Sato, S. Higuchi, and J. Shimoiizaka, in Proceedings of the 19th Annual Meeting of the ChemicalSociety of Japan, 293, 1966.

[45] I. Nakatani, M. Hijikata, and K. Ozawa, "Iron-nitride magnetic fluids prepared by vapor-liquid reaction and their magnetic properties," Journal of Magnetism and Magnetic Materials, vol. 122, no. 1-3, pp. 10-14, 1993.

[46] S. Sun, C. B. Murray, D. Weller, L. Folks, and A. Moser, "Monodisperse FePt nanoparticles and ferromagnetic FePt nanocrystal superlattices," Science, vol. 287, no. 5460, pp. 19891992, 2000.

[47] X.-M. Lin and A. C. S. Samia, "Synthesis, assembly and physical properties of magnetic nanoparticles," Journal of Magnetism and Magnetic Materials, vol. 305, no. 1, pp. 100-109, 2006.

[48] A. H. Lu, E. L. Salabas, and F. Schüth, "Magnetic nanoparticles: synthesis, protection, functionalization, and application," Angewandte Chemie, vol. 46, no. 8, pp. 1222-1244, 2007.

[49] L. Li, Y. Yang, J. Ding, and J. Xue, "Synthesis of magnetite nanooctahedra and their magnetic field-induced two-/threedimensional superstructure," Chemistry of Materials, vol. 22, no. 10, pp. 3183-3191, 2010. 
[50] J. L. C. Huaman, S. Fukao, K. Shinoda, and B. Jeyadevan, "Novel standing Ni-Pt alloy nanocubes," CrystEngComm, vol. 13, no. 10, pp. 3364-3369, 2011.

[51] Y. Li, Q. Zhang, A. V. Nurmikko, and S. Sun, "Enhanced magnetooptical response in dumbbell-like $\mathrm{Ag}-\mathrm{CoFe}_{2} \mathrm{O}_{4}$ nanoparticle pairs," Nano Letters, vol. 5, no. 9, pp. 1689-1692, 2005.

[52] The International Commission on Non-Ionizing Radiation Protection, "Guide-lines for limiting exposure to time-varying electric, magnetic, and electro-magnetic fields (up to $300 \mathrm{GHz}$ )," Health Physics, vol. 74, no. 4, pp. 494-522, 1998.

[53] E. Lima Jr., E. de Biasi, and M. V. Mansilla, "Heat generation in agglomerated ferrite nanoparticles in an alternating magnetic field," Journal of Physics D, vol. 46, no. 4, Article ID 045002, 2013.

[54] S. M. Morgan and R. H. Victora, "Use of square waves incident on magnetic nanoparticles to induce magnetic hyperthermia for therapeutic cancer treatment," Applied Physics Letters, vol. 97, no. 9, Article ID 093705, 2010.

[55] E. L. Verde, G. T. Landi, and M. S. Carriao, "Field dependent transition to the non-linear regime in magnetic hyperthermia experiments: comparison between maghemite, copper, zinc, nickel and cobalt ferrite nanoparticles of similar sizes," AIP Advances, vol. 2, no. 3, Article ID 032120, 23 pages, 2012.

[56] G. T. Landi and A. F. Bakuzis, "On the energy conversion efficiency in magnetic hyperthermia applications: a new perspective to analyze the departure from the linear regime," Journal of Applied Physics, vol. 111, no. 8, Article ID 083915, 2012.

[57] N. A. Usov, S. A. Gudoshnikov, and O. N. Serebryakova, "Properties of dense assemblies of magnetic nanoparticles promising for application in biomedicine," Journal of Superconductivity and Novel Magnetism, vol. 26, no. 4, pp. 1079-1083, 2013.

[58] J. Carrey, B. Mehdaoui, and M. Respaud, "Simple models for dynamic hysteresis loop calculations of magnetic singledomain nanoparticles: application to magnetic hyperthermia optimization," Journal of Applied Physics, vol. 109, no. 8, Article ID 083921, 17 pages, 2011.

[59] Z. P. Mendoza, G. A. Pasquevich, and S. J. Stewart, "Structural and magnetic study of zinc-doped magnetite nanoparticles and ferrofluids for hyperthermia applications," Journal of Physics D, vol. 46, no. 12, Article ID 125006, 2013.

[60] W. F. Brown Jr., "Thermal fluctuations of a single-domain particle," Physical Review, vol. 130, no. 5, pp. 1677-1686, 1963.

[61] H. Mamiya and B. Jeyadevan, "Optimal design of nanomagnets for targeted hyperthermia," Journal of Magnetism and Magnetic Materials, vol. 323, no. 10, pp. 1417-1422, 2011.

[62] D. B. Reeves and J. B. Weaver, "Simulations of magnetic nanoparticle Brownian motion," Journal of Applied Physics, vol. 112, no. 12, Article ID 124311, 6 pages, 2012.

[63] T. Yoshida and K. Enpuku, "Simulation and quantitative clarification of AC susceptibility of magnetic fluid in nonlinear Brownian relaxation region," Japanese Journal of Applied Physics, vol. 48, Article ID 127002, 7 pages, 2009.

[64] H. Mamiya and B. Jeyadevan, "Hyperthermic effects of dissipative structures of magnetic nanoparticles in large alternating magnetic fields," Scientific Reports, vol. 1, article 157, 2011.

[65] N. A. Usov and B. Ya. Liubimov, "Dynamics of magnetic nanoparticle in a viscous liquid: application to magnetic nanoparticle hyperthermia," Journal of Applied Physics, vol. 112, no. 2, Article ID 023901, 11 pages, 2012.

[66] H. Mamiya and B. Jeyadevan, "Formation of non-equilibrium magnetic nanoparticle structures in a large alternating magnetic field and their influence on magnetic hyperthermia treatment,"
IEEE Transactions on Magnetics, vol. 48, no. 11, pp. 3258-3262, 2012.

[67] H. Mamiya and B. Jeyadevan, "Magnetic hysteresis loop in a superparamagneticstate," in press. IEEE Transactions on Magnetics.

[68] W. Andrä, C. G. D’Ambly, R. Hergt, I. Hilger, and W. A. Kaiser, "Temperature distribution as function of time around a small spherical heat source of local magnetic hyperthermia," Journal of Magnetism and Magnetic Materials, vol. 194, no. 1, pp. 197203, 1999.

[69] C. W. Song, "Effect of local hyperthermia on blood flow and microenvironment: a review," Cancer Research, vol. 44, no. 10, supplement, pp. 4721s-4730s, 1984.

[70] T. Hasegawa, R. Kudaka, K. Saito et al., Bulletin of Suzuka University of Medical Science, vol. 11, pp. 58-64, 2004.

[71] J. Bohnert and O. Dössel, "Simulations of temperature increase due to time varying magnetic fields up to $100 \mathrm{kHz}$," in Proceedings of the 5th European Conference of the International Federation for Medical and Biological Engineering, vol. 37 of IFMBE Proceedings, pp. 303-306, 2012.

[72] H. Mamiya, "Magnetic response of nanoparticles to AC magnetic fields and targeted thermotherapy," Materials Integration, vol. 25, pp. 11-23, 2012.

[73] T. Kobayashi, "Cancer hyperthermia using magnetic nanoparticles," Biotechnology Journal, vol. 6, no. 11, pp. 1342-1347, 2011.

[74] B. Thiesen and A. Jordan, "Clinical applications of magnetic nanoparticles for hyperthermia," International Journal of Hyperthermia, vol. 24, no. 6, pp. 467-474, 2008. 

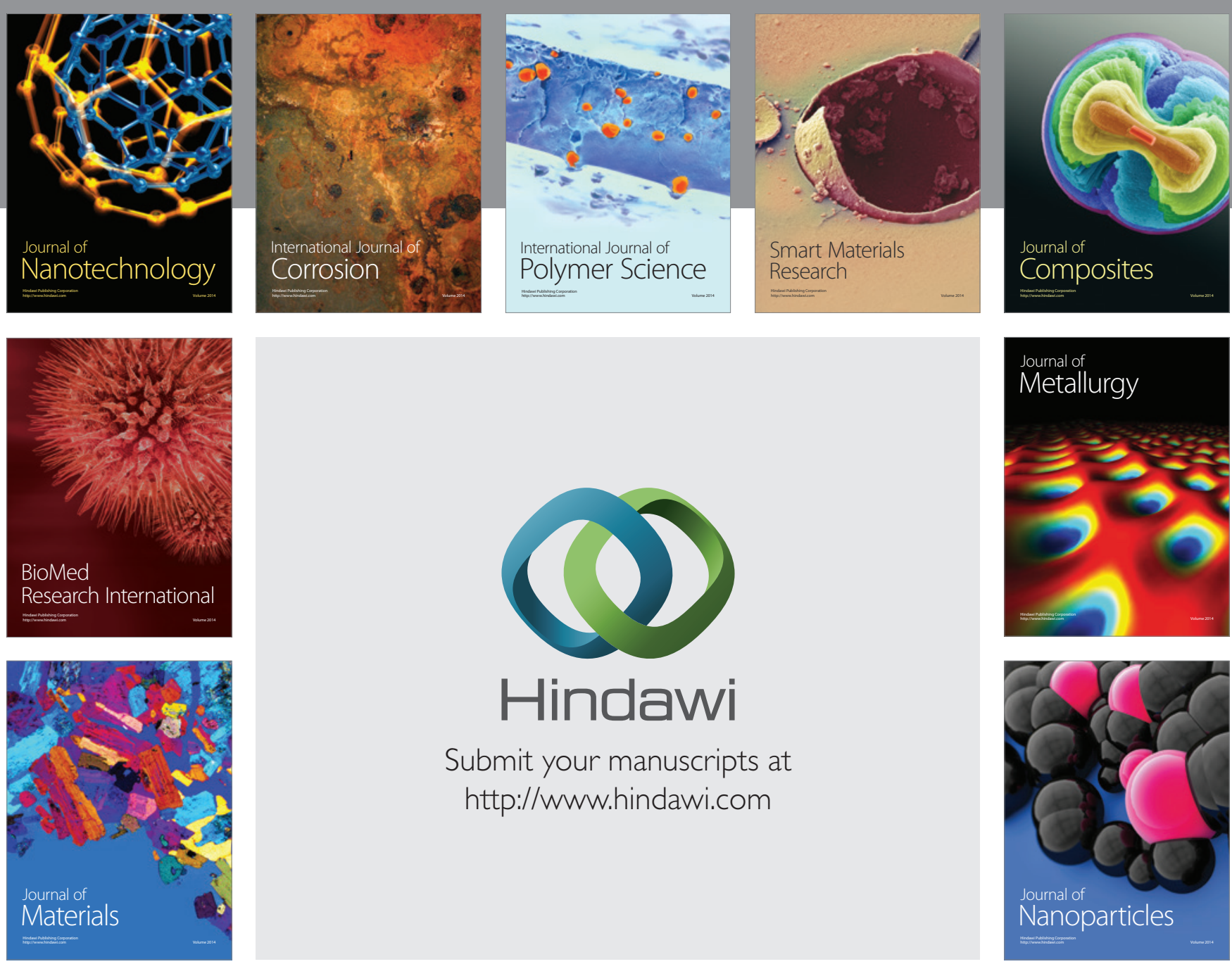

Submit your manuscripts at http://www.hindawi.com
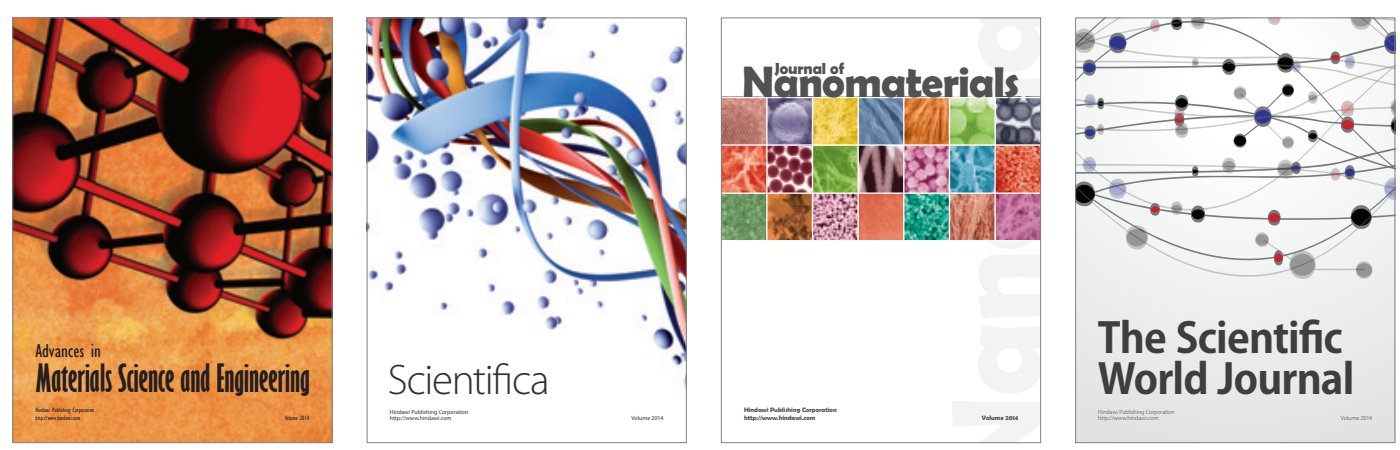

\section{The Scientific World Journal}
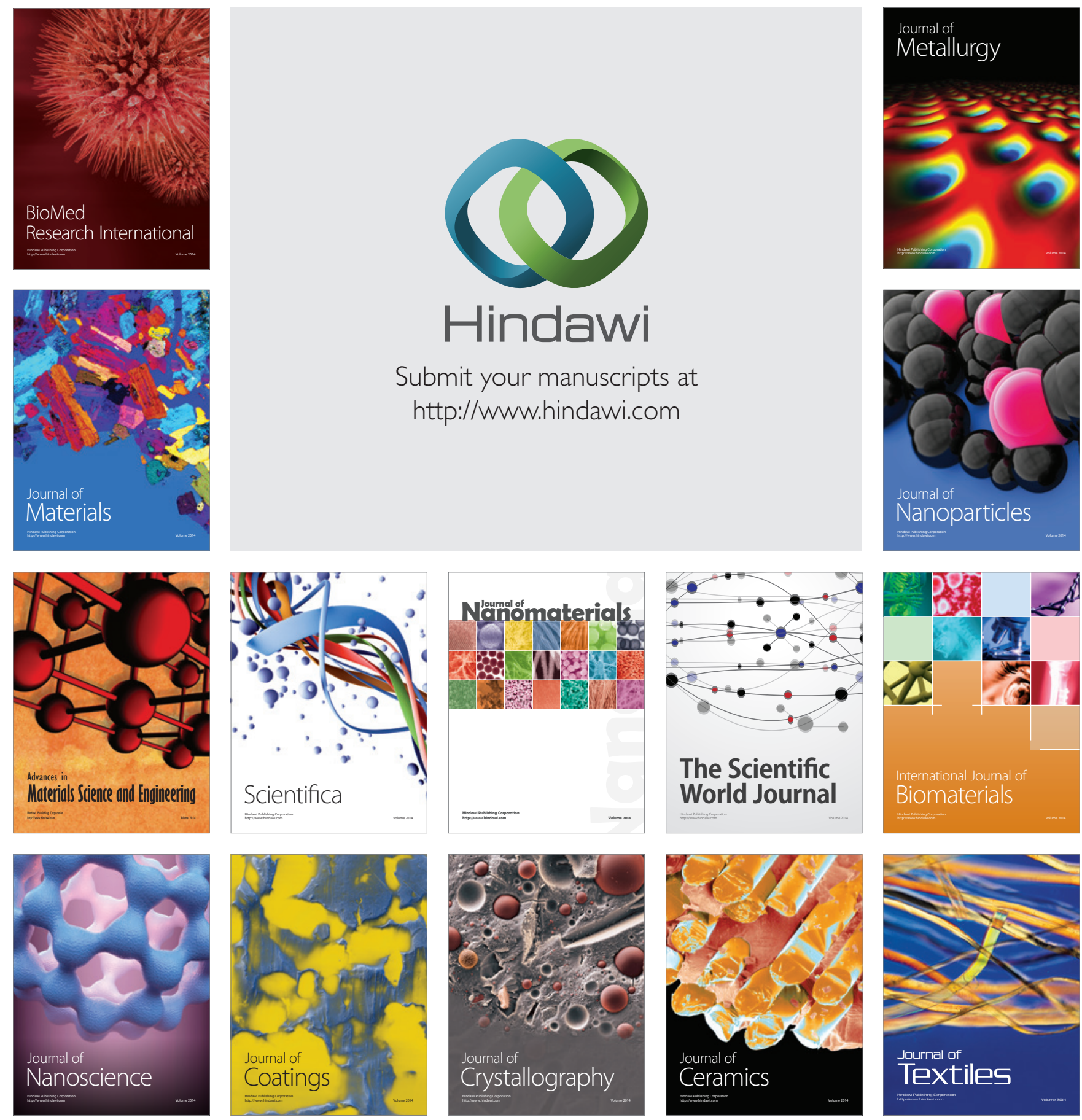